\title{
Depletion of CD206+ Tumour Macrophages via a Peptide Targeted Star-Shaped Polyglutamate Inhibits Tumourigenesis and Metastatic Dissemination in Mouse Breast Cancer Models
}

\section{Anni Lepland}

Laboratory of Precision and Nanomedicine, Institute of Biomedicine and Translational Medicine, University of Tartu https://orcid.org/0000-0002-6448-4847

\section{Alessio Malfanti}

Polymer Therapeutics Laboratory, Prince Felipe Research Center

\section{Uku Haljasorg}

Molecular Pathology Research Group, Institute of Biomedicine and Translational Medicine, University of Tartu https://orcid.org/0000-0002-1541-6020

\section{Eliana Asciutto}

School of Science and Technology, National University of San Martin (UNSAM) ICIFI and CONICET.

\section{Monica Pickholz}

Departamento de Física, Facultad de Ciencias Exactas y Naturales, Universidad de Buenos Aires; Instituto de Física de Buenos Aires (IFIBA), CONICET-Universidad de Buenos Aires

\section{Mauro Bringas}

Departamento de Química Inorgánica, Analítica y Química Física, Facultad de Ciencias Exactas y Naturales, Universidad de Buenos Aires; Fundación Instituto L

\section{Snežana Đorđević}

Polymer Therapeutics Laboratory, Prince Felipe Research Center

\section{Liis Salumäe}

Tartu University Hospital, Pathology department

\section{Pärt Peterson}

Molecular Pathology Research Group, Institute of Biomedicine and Translational Medicine, University of Tartu https://orcid.org/0000-0001-6755-791X

\section{Tambet Teesalu}

Laboratory of Precision and Nanomedicine, Institute of Biomedicine and Translational Medicine, University of Tartu; Centre for Nanomedicine and Department of Cell, Molecular and Developmental Biolog

\section{María Vicent}

Polymer Therapeutics Laboratory, Prince Felipe Research Center https://orcid.org/0000-0001-7771- 
Pablo Scodeller ( $\sim$ pablo.david.scodeller@ut.ee )

Laboratory of Precision and Nanomedicine, Institute of Biomedicine and Translational Medicine, University of Tartu

\section{Research Article}

Keywords: polymer-drug nanoconjugates, targeting peptide, CD206, tumour-associated macrophages, triple negative breast cancer, pulmonary metastasis, immunomodulation

Posted Date: December 30th, 2021

DOI: https://doi.org/10.21203/rs.3.rs-839856/v2

License: (c) (i) This work is licensed under a Creative Commons Attribution 4.0 International License.

Read Full License 
Anni Lepland ${ }^{¥, 1}$, Alessio Malfanti ${ }^{¥, 2}$, Uku Haljasorg ${ }^{3}$, Eliana K. Asciutto ${ }^{4}$, Monica Pickholz $^{5,6}$, Mauro Bringas ${ }^{7,8}$, Snežana Đorđević ${ }^{2}$, Liis Salumäe ${ }^{9}$, Pärt Peterson ${ }^{3}$, Tambet Teesalu $^{\psi, 1,10}$, María J. Vicent ${ }^{\psi, 2}$, Pablo Scodeller ${ }^{\psi, 1}$

$¥:$ equal contribution

$\psi$ : equal contribution

${ }^{1}$ Laboratory of Precision and Nanomedicine, Institute of Biomedicine and Translational Medicine, University of Tartu, Ravila 14B, Tartu, 50411, Estonia

${ }^{2}$ Polymer Therapeutics Laboratory, Prince Felipe Research Centre, Av. Eduardo Primo Yúfera 3, Valencia 46012, Spain

${ }^{3}$ Molecular Pathology Research Group, Institute of Biomedicine and Translational Medicine, University of Tartu, Ravila 19, Tartu, 50412, Estonia

${ }^{4}$ School of Science and Technology, National University of San Martin (UNSAM) ICIFI and CONICET. Buenos Aires, Argentina.

${ }^{5}$ Departamento de Física, Facultad de Ciencias Exactas y Naturales, Universidad de Buenos Aires, Buenos Aires

${ }^{6}$ Instituto de Física de Buenos Aires (IFIBA), CONICET-Universidad de Buenos Aires, Buenos Aires, Argentina

${ }^{7}$ Departamento de Química Inorgánica, Analítica y Química Física, Facultad de Ciencias Exactas y Naturales, Universidad de Buenos Aires, Ciudad de Buenos Aires, C1428EHA, Argentina

${ }^{8}$ Fundación Instituto Leloir, Instituto de Investigaciones Bioquímicas de Buenos Aires (IIBBACONICET), C1405BWE Ciudad Autónoma de Buenos Aires, Buenos Aires, Argentina.

${ }^{9}$ Tartu University Hospital, Pathology department, Puusepa 8, 50406, Estonia

${ }^{10}$ Centre for Nanomedicine and Department of Cell, Molecular and Developmental Biology, University of California, Santa Barbara, 93106, CA, USA 


\section{ABSTRACT}

38 Although many studies have explored the depletion of tumour-associated macrophages

39 (TAMs) as a therapeutic strategy for solid tumours, currently available compounds suffer from 40 poor efficacy and dose-limiting side effects. Here, we developed a novel TAM-depleting agent 41 ("OximUNO") that specifically targets CD206 ${ }^{+}$TAMs and demonstrated efficacy in triple 42 negative breast cancer (TNBC) mouse models. OximUNO comprises a star-shaped 43 polyglutamate (St-PGA) decorated with the CD206-targeting peptide mUNO that carries the 44 chemotherapeutic drug doxorubicin (DOX). In TNBC models, a fluorescently-labelled 45 mUNO-decorated St-PGA homed to CD206 ${ }^{+}$TAMs within primary lesions and metastases. 46 OximUNO exhibited no acute liver or kidney toxicity in vivo. Treatment with OximUNO 47 reduced the progression of primary tumour lesions and pulmonary metastases, significantly 48 diminished the number of $\mathrm{CD}_{206^{+}}$TAMs and increased the CD8/FOXP3 expression ratio 49 (demonstrating immunostimulation). Our findings suggest the potential benefit of OximUNO 50 as a TAM-depleting agent for TNBC treatment. Importantly, our studies also represent the first 51 report of a peptide-targeted St-PGA as a targeted therapeutic nanoconjugate.

53 Keywords: polymer-drug nanoconjugates, targeting peptide, CD206, tumour-associated macrophages, triple negative breast cancer, pulmonary metastasis, immunomodulation

\section{INTRODUCTION}

Triple negative breast cancer (TNBC), defined by the lack of the expression of the oestrogen receptor (ER), progesterone receptor (PR), and human epidermal growth factor receptor 2 $(\text { HER2) })^{1,2}$, represents an aggressive breast cancer subtype with poor prognosis ${ }^{3}$ that comprises up to $20 \%$ of all breast cancer cases ${ }^{3,4}$. Interfering with immune checkpoints signalling (e.g. through the modulation of programmed cell death 1 (PD-1) and its ligand (PD-L1)) represents an alternative treatment strategy for several cancers and is currently being employed in combination with chemotherapy as a neoadjuvant or adjuvant treatment ${ }^{5-8}$. The U.S. Food and Drug Administration (FDA) recently granted accelerated approval for a combination of a PDL1 blocking antibody (atezolizumab, Tecentriq ${ }^{\circledR}$ ) and nab-paclitaxel (Abraxane $\left.{ }^{\circledR}\right)^{9}$ as a first-

67 line treatment for unresectable locally advanced or metastatic $\mathrm{TNBC}^{10}$. While promising 68 clinical results have resulted, this combinatorial treatment approach suffers from significant 69 obstacles, including the problematic identification and heterogeneity of PD-L1 expression in 
70 patients $^{11}$, the limited applicability to PD-L1 positive TNBC patients (only 20-42\% of 71 cases) $)^{12,13}$, and the induction of severe side effects (e.g., neutropenia, peripheral neuropathy, 72 and colitis) ${ }^{10,14,15}$. Other immune checkpoint inhibitors (ICIs), including the cytotoxic T 73 lymphocyte-associated antigen 4 (CTLA-4) blockers ipilimumab and tremelimumab, are 74 currently under evaluation for TNBC treatment in combination with other drugs (clinical trial 75 identifiers: NCT03606967, NCT02983045); however, anti-CTLA-4 treatments induce severe 76 side effects such as endocrinopathies, myopathy, enterocolitis, and hepatitis ${ }^{16-19}$, which narrow 77 their use. Overall, the limited success of alternative treatment options for TNBC has maintained 78 chemotherapy as the standard of care for most patients ${ }^{20}$.

79 The anthracycline drug doxorubicin (DOX), which presents high off-target effects such as 80 cardiotoxicity $^{21,22}$, represents a frequently employed chemotherapeutic for TNBC; however, 81 disease relapse and metastatic development have also been associated with DOX treatment ${ }^{23}$. 82 M2 (anti-inflammatory)-polarised tumour-associated macrophages (TAMs) ${ }^{24}$ found within 83 both primary and metastatic tumour lesions mediate both events ${ }^{25}$; furthermore, TAMs 84 represent the main executioners of tumour progression, immunosuppression and invasion ${ }^{24-29}$, 85 and their presence correlates with inadequate therapeutic response and poor prognosis ${ }^{25}$. 86 Recent efforts have focused on eliminating TAMs, and several ongoing clinical trials are 87 currently evaluating TAM depletion in combination with treatments such as ICIs ${ }^{30}$. The current 88 clinical-stage gold standard for TAM depletion relies on agents that block colony stimulating 89 factor 1 (CSF1) or its receptor CSF1R, such as the small molecule CSF1R inhibitor PLX339731; 90 however, microglia also expresses CSF1R ${ }^{32}$, the inhibition of CSF1R with PLX5622 impacts 91 M1 macrophages ${ }^{33}$, and PLX3397 treatment causes oedema ${ }^{34}$. Clinical data suggests that anti92 CSF1R antibodies induce a modest effect ${ }^{35,36}$ and cause severe side effects that include 93 haematological toxicities ${ }^{35}$ and hepatotoxicity by targeting Kupfer cells ${ }^{35,36}$. Overall, these 94 findings highlight the overwhelming need for new TAM-depletion strategies.

95 Notably, both perivascular TAMs associated with disease relapse and therapeutic resistance ${ }^{24}$ 96 and metastasis-associated macrophages ${ }^{37}$ express the mannose receptor (CD206/MRC1). 97 Perivascular TAMs employ CD206 to navigate the surrounding collagen-dense stroma ${ }^{38}$, 98 which favours tumour progression ${ }^{39,40}$.

99 For the first time, we report the effects of depleting the CD206 ${ }^{+}$subpopulation of TAMs in 100 metastatic TNBC mouse models through the use of a targeting agent (the mUNO peptide) for 101 a CD206 site different from the mannose-binding site ${ }^{41-44}$. Previous studies have employed 102 mannose to target CD206; however, mannose has other receptors besides CD206 $6^{45,46}$. 
103 We decorated a three-arm branched biodegradable multivalent polyanion with a defined 104 negative charge and nanometre-size hydrodynamic radius (star-shaped polyglutamate or St105 PGA) with mUNO peptide to function as a targeted delivery platform for a chemotherapeutic 106 agent (DOX) conjugated through a bioresponsive linker. St-PGA-DOX-mUNO (referred to as 107 OximUNO) efficiently depleted CD206 ${ }^{+}$TAMs, relieved immunosuppression in the tumour 108 microenvironment (TME) and limited metastasis/tumour growth, thereby supporting 109 OximUNO as an alternative TAM depletion strategy.

110 Most importantly, this study represents the first described combination of two reported 111 technologies - the St-PGA nanocarrier and the mUNO targeting peptide. Overall, this

112 OximUNO proof-of-concept demonstrates the potential of the peptide-targeted St-PGA 113 nanosystem. Our studies lay a foundation for future work using this nanosystem to target other 114 receptors efficiently by changing the targeting peptide.

\section{RESULTS}

\section{Design and structural modelling of St-PGA-OG-mUNO}

119 To characterise and explore the function of OximUNO, we first developed an mUNO-targeted 120 St-PGA labelled with the Oregon Green (OG) fluorescent dye (referred to as St-PGA-OG121 mUNO) (Fig. 1A, Scheme S1). We conjugated OG to St-PGA using an amide linker to allow 122 in vitro or in vivo tracking and coupled mUNO through a disulphide bond formed between the 123 free cysteine of mUNO and a pyridyldithiol linker on St-PGA. We previously demonstrated 124 that mUNO conjugated to polymeric nanostructures through the cysteine thiol group preserves 125 CD206 binding ${ }^{42}$. To evaluate the structure and dye loading, we analysed St-PGA-OG-mUNO 126 and St-PGA-OG using nuclear magnetic resonance (NMR) and UV-Vis analyses (Fig. S1).

127 Dynamic light scattering (DLS) analysis demonstrated that St-PGA-OG-mUNO and St-PGA128 OG displayed similar hydrodynamic diameters of 6.8 and $5.9 \mathrm{~nm}$, respectively (Fig. 1B, C), 129 while both nanoconjugates exhibited highly negative charges $(-42 \mathrm{mV}$ and $-48 \mathrm{mV}$, 130 respectively) as shown by Zeta potential analysis (Fig. 1B); an expected result given the 131 glutamic acid nature of the polymer carrier. Analysis of mUNO loading (Fig. 1B) indicated the 132 presence of approximately seven mUNO peptides in St-PGA-mUNO nanoconjugate, which 133 would allow multivalent receptor binding.

134 We next assessed the structure of unlabelled and untargeted St-PGA in water using molecular 135 dynamics (MD) simulations to access information at the atomic scale. We assumed an initial 
136 helical conformation for the three PGA chains. The studied system consisted of a fully hydrated 137 St-PGA and the $\mathrm{Na}^{+}$counterions ( 920,000 atoms) and was built after initial minimisation 138 under vacuum conditions. We simulated 50 ns of the entire St-PGA macromolecule, with Fig. 139 1D displaying a snapshot corresponding to the last step of the simulation. Averaging the 140 gyration radius over the last $25 \mathrm{~ns}$ of the simulation run provided a value of $5.6 \pm 0.3 \mathrm{~nm}$, which 141 lies in the same order of magnitude as the results from DLS analysis and suggests a lack of 142 aggregation of both St-PGA-OG-mUNO and St-PGA-OG in PBS. A video simulation (Video 143 S1) suggested that the three PGA chains remain in an extended conformation throughout the 144 simulation and do not show any intra- or inter-molecular interaction, suggesting that the mUNO 145 peptides linked to St-PGA will not interfere with each other.

146 To investigate if mUNO can engage with the CD206 receptor when grafted onto St-PGA, we 147 modelled the structure and mobility of St-PGA-mUNO using computational analysis. To attain 148 a computationally feasible system, we simulated only single branches of St-PGA-mUNO. We 149 placed three equidistant mUNO peptides on a PGA single branch and fully solvated the system.

150 We observed that three mUNO peptides remained exposed to the solution available for receptor 151 binding (Fig. 1E). The rotation of mUNO around PGA, tracked by the angle formed by a 152 proline aromatic carbon within mUNO (Fig. S2, green sphere), a pyridyldithiol linker nitrogen 153 (Fig. S2, blue sphere), and a glutamic acid aromatic carbon (Fig. S2, light blue sphere) revealed 154 angles between $50^{\circ}$ and $180^{\circ}$ (Fig. 1F). This value supports the ability of mUNO peptides to 155 interact with their receptor ${ }^{43}$. Comparisons with an undecorated PGA branch demonstrated the 156 minimal alterations of secondary structure dynamics in the presence of mUNO peptides 157 turning alpha helices (Fig. 1G, green) into random coils (Fig. 1G, brown) at regions where they 158 are placed; however, the PGA chain structure remained mainly helical except in the middle, 159 where a slight kink formed (Fig. 1G). 
A

St-PGA- $\underline{\text { OG-mUNO }}$
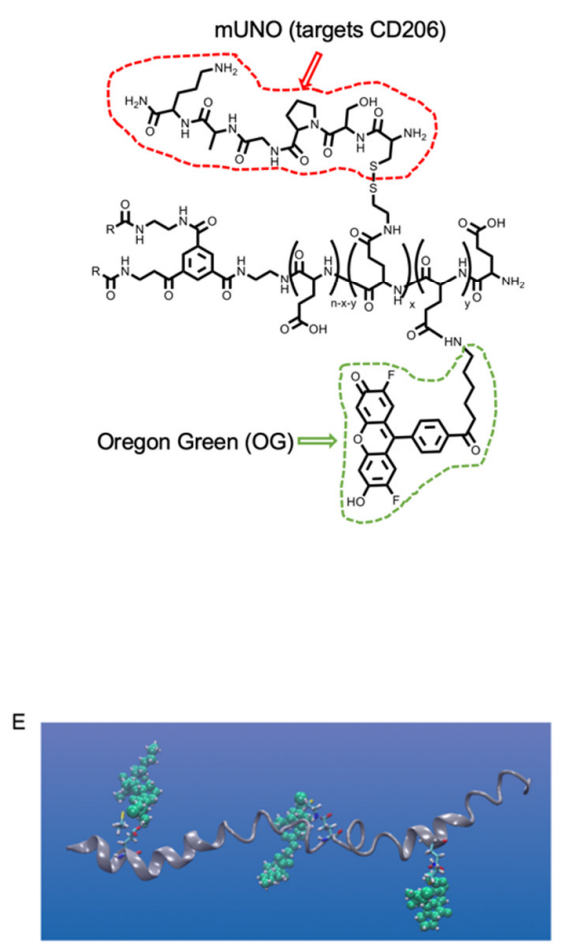

B

\begin{tabular}{lcccc} 
Compound & $\begin{array}{c}\% \text { wt } \\
\text { OG }\end{array}$ & $\begin{array}{c}\text { \% mol } \\
\text { mUNO }\end{array}$ & Size $(\mathrm{d}, \mathrm{nm})$ & $\begin{array}{c}\text { Zeta potential } \\
(\mathrm{mV})\end{array}$ \\
\hline $\begin{array}{l}\text { St-PGA-OG- } \\
\text { mUNO }\end{array}$ & 0.776 & 5.00 & $6.82 \pm 1.80$ & $-41.6 \pm 5.04$ \\
\hline St-PGA-OG & 0.844 & - & $5.93 \pm 0.68$ & $-47.6 \pm 6.13$
\end{tabular}

C
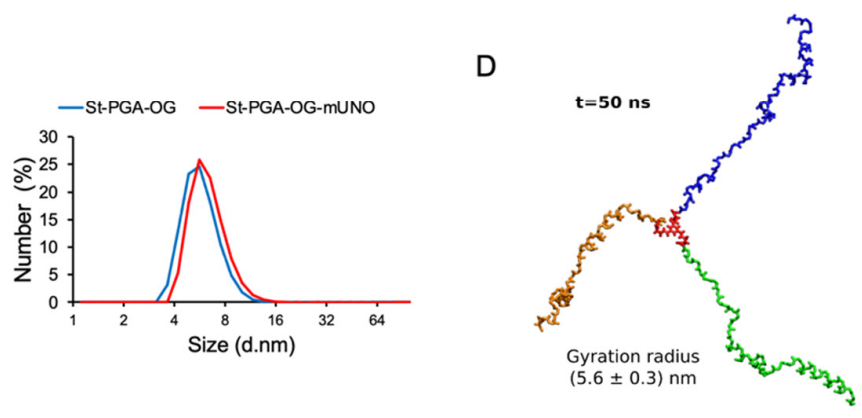

$\mathrm{F}$

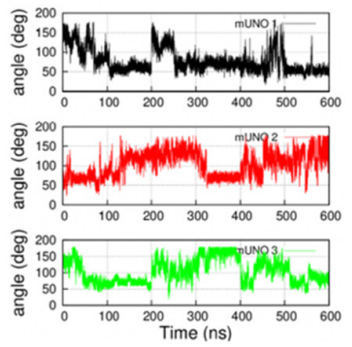

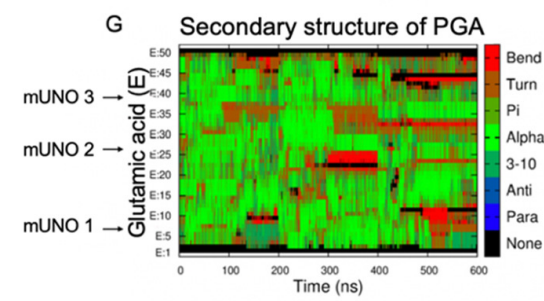

161

Figure 1. Design and analysis of mUNO-targeted St-PGA. (A) Representative stru ture of St-PGA de orated with mUNO peptides (red) and OG (green). (B) Table detailing OG loading, mUNO loading, size (as measured by DLS), and harge (as measured by Zeta potential analysis). (C) DLS graph demonstrating uniform size for both St-PGA-OG-mUNO and St-PGA-OG. (D) A snapshot of modelled St-PGA stru ture in water and $\mathrm{Na}^{+}$ ounterions at the last stage of the simulation $(50 \mathrm{~ns})$, displaying the three arms in different olours for visual Clarity. The average gyration radius was $5.6 \pm 0.3 \mathrm{~nm}, \mathrm{t}$ shows time in $\mathrm{ns}$. (E) Representative MD snapshot of a single St-PGA-mUNO bran $\square$ $\sqsubset$ ontaining three equidistant mUNO peptides. Green spheres represent mUNO and a $\mathrm{Li} \sqsubset$ ori $\sqsubset$ representation shows the linker. $(\mathbf{F})$ mUNO rotation around the PGA hain for ea $h$ of the three peptides (bla $\mathrm{k}$, red, and green lines). (G) PGA hain se $\_$ondary stru ture evolution, where red and brown regions show how mUNO perturbs the $\pitchfork$ hain stru ture, turning alpha-heli $\llbracket$ es into random $₫$ oils.

Altogether, St-PGA-OG-mUNO and St-PGA-OG nanoconjugates possessed similar sizes by DLS, highly negative charges, and, according to simulations, displayed their three arms in an extended open structure. Our simulation analyses demonstrated that mUNO peptides induced a minimal effect on PGA structure and rotated around the PGA chain with considerable freedom. Overall, these findings suggest St-PGA-mUNO as a suitable platform for CD206 targeting. 
180 St-PGA-OG-mUNO targets CD206 ${ }^{+}$TAMs and displays low Liver accumulation

181 We next evaluated the potential of St-PGA-OG-mUNO to target CD206 ${ }^{+}$TAMs in two 182 different TNBC models - an orthotopic TNBC model and an experimental metastasis of TNBC 183 model induced by intravenous (i.v.) injection of 4T1 cells. We administered St-PGA-OG184 mUNO or St-PGA-OG intraperitoneally (i.p.), allowed circulation for 6 h, and then analysed 185 tumour homing using confocal fluorescence microscopy. Our previous study provided the 186 rationale for the i.p. administration route, where we demonstrated that the i.p. administered 187 mUNO peptide exhibited a substantially longer half-life than intravenously (i.v.) administered 188 mUNO in the same mice (same strain, sex and age) used in this study ${ }^{42}$.

189 In the orthotopic TNBC model, we observed a high colocalisation of OG/CD206 (Fig. 2A, 190 yellow signal) with St-PGA-OG-mUNO but a much lower colocalisation of OG/CD206 with 191 non-targeted St-PGA-OG (Fig. 2B) (0.57 and 0.21, respectively (Fig. 2M)). We observed a low 192 level of accumulation of St-PGA-OG-mUNO or St-PGA-OG in the liver (Fig. S3A, B). We 193 employed confocal image acquisition parameters throughout this study to visualise CD206 in 194 the tumour without signal saturation. Given the higher levels of CD206 in the tumour, imaging 195 with associated settings provides low CD206 visualisation in the liver. Using a higher image 196 intensity, we observed the expected CD206 signal in the liver (as expected from Kupfer cells 197 and sinusoid vessels) (Fig. S4A) and a saturated CD206 signal in the tumour (Fig. S4B).

198 Immunostaining for endogenous mouse $\operatorname{IgG}$ in the tumour and the liver indicated the leaky 199 nature of the tumour vasculature (Fig. S5A) compared to the liver vasculature (Fig. S5B) in the 200 4T1 model. A leaky tumour vasculature favours the hypothesis that St-PGA-OG-mUNO has a 201 more extended (both in time and space) access to CD206 in the tumour than in the liver. We 202 speculate that the leaky tumour vasculature combined with lower CD206 expression in the liver 203 than the tumour explains the low liver accumulation of St-PGA-OG-mUNO. St-PGA-OG204 mUNO did not accumulate in the lungs (Fig. S6A) or spleen (Fig. S6B); however, we did 205 observe some accumulation in the sentinel lymph node (SLN) (Fig. S6C) and the kidneys (Fig. 206 S6D). Of note, the observed kidney signal agrees with our prior studies that demonstrated the 207 renal excretion of St-PGA ${ }^{47}$.

208 Importantly, we did not detect homing to M1 macrophages $\left(\mathrm{CD} 86^{+}\right)$or dendritic cells $\left(\mathrm{CD} 11 \mathrm{c}^{+}\right.$, 209 DCs) with St-PGA-OG-mUNO (Fig. 2C, E) or with St-PGA-OG (Fig. 2D, F). In the 210 experimental metastasis of TNBC model, most of the cellular signal of St-PGA-OG-mUNO 211 associated with CD206 ${ }^{+}$TAMs (Fig. 2G, yellow signal) when compared to St-PGA-OG (Fig. 
212 2H) (OG/CD206 colocalisation 0.42 and 0.14, respectively, Fig. 2N). In this model, we also 213 observed no colocalisation between OG and CD86 (M1 macrophages) (Fig. 2I, J) or OG and 214 CD11c (DCs) (Fig. 2K, L) and the observed liver accumulation of St-PGA-OG-mUNO or St215 PGA-OG was low (Fig. S7).

216 One of the rationales behind the design of OximUNO was to increase mUNO targeting through 217 increased avidity and plasma half-life. To evaluate these aspects, we compared the homing of 218 St-PGA-OG-mUNO with a monomeric, carboxyfluorescein-labelled mUNO peptide (FAM219 mUNO). We note that even given the different nature of the fluorescent labels (OG on St-PGA220 OG-mUNO and fluorescein on FAM-mUNO), we did not use their native fluorescence as a 221 readout; instead, we used an antibody that recognises both FAM and OG; therefore, we do not 222 expect biases from potential differences in FAM and OG emissions.

223 We discovered that St-PGA-OG-mUNO (Fig. S8A) displayed significantly higher OG/CD206 224 colocalisation than for FAM/CD206 with FAM-mUNO (Fig. S8B) at $6 \mathrm{~h}(0.74$ vs. 0.21, 225 respectively (Fig. 2O)). Additionally, we found that the OG/FAM mean signal per CD206 ${ }^{+}$cell 226 was five times higher for St-PGA-OG-mUNO than FAM-mUNO (Fig. 2P). These findings 227 suggest that conjugating mUNO to the St-PGA backbone greatly improved receptor binding. 228 Plasma half-life analysis for i.p. administered St-PGA-OG-mUNO revealed a $4.5 \mathrm{~h}$ half-life 229 (Fig. 2Q), a value over two times longer than that observed after the i.p. administration of 230 FAM-mUNO in our previous study ${ }^{42}$. Overall, this finding suggests that conjugating onto St231 PGA increased the plasma half-life of mUNO peptide, a desirable feature that will improve in 232 vivo ligand targeting. 


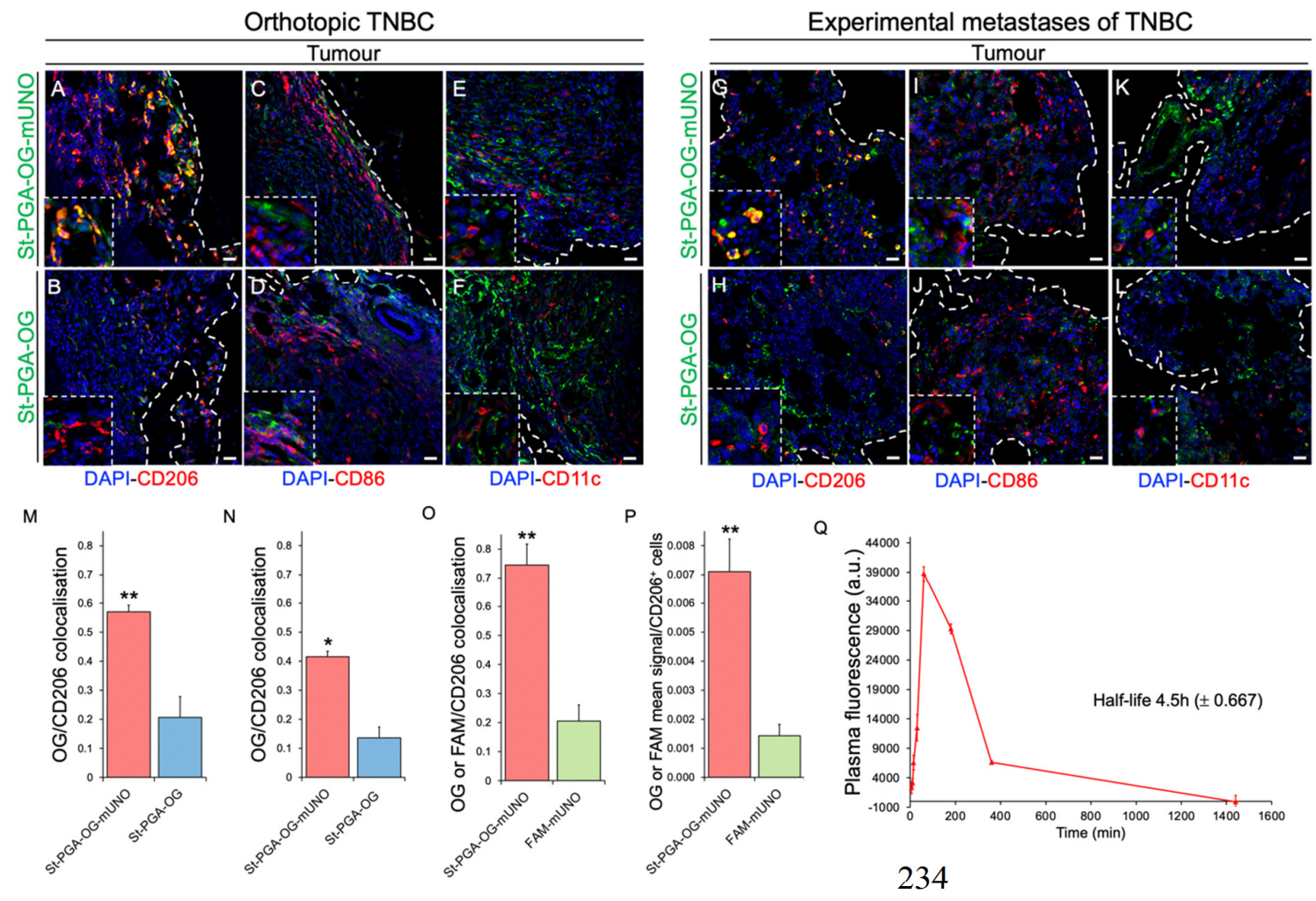

235 Figure 2. St-PGA-OG-mUNO targets $\mathrm{CD206}^{+}$TAMs in models of orthotopic TNBC and experimental 236 metastasis of TNBC and displays an extended plasma half-life. Homing studies with i.p. administered St-PGA237 OG-mUNO (0.41 mg/0.5mL of PBS) or St-PGA-OG (0.35 mg/0.5mL of PBS), after $6 \mathrm{~h}$ of circulation. $\mathrm{N}=3$ for 238 orthotopic TNBC and N=2 for experimental metastasis of TNBC. (A-F) Homing in orthotopic TNBC. (A) St239 PGA-OG-mUNO displayed high colocalisation between OG and CD206 (yellow signal), whereas (B) St-PGA240 OG displayed minimal colocalisation. St-PGA-OG-mUNO and St-PGA-OG did not show any homing to CD86 ${ }^{+}$ 241 cells (M1 macrophages) (C, D) nor CD11 c cells (DCs) (E, F). (G-L) Homing study in the experimental metastasis 242 of TNBC. (G) St-PGA-OG-mUNO displayed high colocalisation with OG and CD206 (yellow signal), whereas 243 (H) St-PGA-OG showed minimal colocalisation. St-PGA-OG-mUNO and St-PGA-OG did not show any homing 244 to CD86 $^{+}$cells (M1 macrophages) (I, J) or CD11 c ${ }^{+}$cells (DCs) (K, L). Scale bars represent $20 \mu \mathrm{m}$. (M) Graphs 245 depicting the quantification of CD206 and OG colocalisation in the orthotopic TNBC and (N) the experimental 246 metastasis of TNBC. (O) Quantification of colocalisation analysis for St-PGA-OG-mUNO or FAM-mUNO with 247 CD206 homing after $6 \mathrm{~h}$ of circulation, $\mathrm{N}=2$ (30nmoles in OG and FAM, respectively). Colocalisation was 248 quantified using the Fiji programme and Pearson's coefficient (for more information see Materials and methods). 249 (P) Mean OG/FAM signal per CD206 ${ }^{+}$cell analysed using the ImageJ programme. (Q) Plasma fluorescence (in 250 the green channel) of i.p. administered St-PGA-OG-mUNO (dose 15nmoles in OG) in healthy Balb/c mice (N=3). 251 Error bars represent the standard error (SE) of the mean.

253 Administration of a higher dose of both nanoconjugates $(0.82 \mathrm{mg} / 0.5 \mathrm{~mL}$ St-PGA-OG-mUNO 254 and $0.7 \mathrm{mg} / 0.5 \mathrm{~mL}$ St-PGA-OG) resulted in high CD206 ${ }^{+}$TAM targeting (Fig. S9A) albeit at 
the expense of higher liver accumulation (Fig. S9C). For this reason, we employed lower nanoconjugate doses $(0.41 \mathrm{mg} / 0.5 \mathrm{~mL}$ and $0.35 \mathrm{mg} / 0.5 \mathrm{~mL})$ for subsequent studies.

We next compared tumour homing of St-PGA-OG-mUNO to that of a therapeutic monoclonal antibody by i.v. injecting anti-PDL1 in orthotopic 4T1 tumour-bearing mice and allowing circulation for $24 \mathrm{~h}$. We observed that administered anti-PDL1 accumulated in the tumour rim (Fig. S10A, TR) but not in the tumour core (Fig. S10A, TC) even given expression of the receptor (PDL1) in the tumour core (Fig. S10B, TC). The observed accumulation of St-PGAOG-mUNO in the tumour core (Fig. S10C) and receptor colocalisation (Fig. S10D), supported the implementation of our platform as an efficient alternative to antibody-based therapies such as anti-PDL1 or antibody-drug conjugates.

Overall, we demonstrated that St-PGA-OG-mUNO, homes to CD206 ${ }^{+}$TAMs in both orthotopic and experimental metastasis of TNBC models with no significant accumulation in the liver. We also established that St-PGA-OG-mUNO does not target M1 macrophages or DCs in the tumour, thereby providing evidence of high specificity for CD206 ${ }^{+}$TAMs.

\section{OximUNO enhances the in vitro cytotoxicity of DOX on M2 macrophages}

St-PGA displays a large surface with multiple sites available for the conjugation of proapoptotic or cytotoxic cargoes via bioresponsive polymer-drug linkers ${ }^{48,49}$. To selectively deplete CD206 ${ }^{+}$TAMs, we conjugated an apoptotic chemotherapeutic agent (DOX) to StPGA-mUNO to form St-PGA-DOX-mUNO (designated “OximUNO”) (Fig. 3A, Scheme S2). We conjugated DOX to St-PGA-mUNO using a hydrazone bond ${ }^{48}$ to allow for site-specific drug release in the acidic milieu of the endosomes/lysosomes ${ }^{48,50}$.

278 To evaluate the effect of mUNO targeting, we included St-PGA-DOX as an untargeted control.

279 We employed ${ }^{1} \mathrm{H}$ NMR and UV-Vis analyses to evaluate the chemical identity of nanoconjugates (Fig. S11A, B).

281 OximUNO displayed DOX and mUNO loadings of $\sim 10 \%$ and $\sim 4 \%$ in weight, respectively, 282 corresponding to around four DOX and seven mUNO molecules for every OximUNO. 283 OximUNO exhibited a size of $\sim 40 \mathrm{~nm}$ and a highly negative surface charge of $-40 \mathrm{mV}$ (Fig. $2843 \mathrm{~B}, \mathrm{C})$. We obtained similar DOX loading, size by DLS, and surface charge values for St-PGA285 DOX (Fig. 3B, C).

286 The $\mathrm{pH}$-sensitive hydrazone linker and the intrinsic biodegradability of St-PGA by lysosomal 287 protease cathepsin B are expected to secure DOX release from OximUNO after cell 
internalisation $^{51}$. Hence, we studied DOX release kinetics from OximUNO in the presence of acidic $\mathrm{pH}(\mathrm{pH} 5)$ and cathepsin $\mathrm{B}$ using liquid chromatography-mass spectrometry (LC-MS, Fig. S12A-G). As we aimed for the i.p. administration of OximUNO, we assessed DOX release in intraperitoneal fluid (i.p. fluid) (Fig. 3D). At pH 5, we observed a sustained DOX release in the first $8 \mathrm{~h}$ (reaching a plateau at 15\%), thereby demonstrating the suitability for endolysosomal drug delivery. DOX release in the presence of cathepsin B displayed comparable values in the first $8 \mathrm{~h}(\sim 13 \%)$, followed by a plateau and a reduced rate in the following hours ( $13 \%$ cumulative release at $72 \mathrm{~h}$ ). Importantly, OximUNO exhibited negligible drug release in both physiological conditions evaluated (PBS and i.p. fluid) (Fig. 3D). We next evaluated the in vitro cytotoxicity of OximUNO and St-PGA-DOX in primary human blood monocyte-derived M2 and M1 macrophages. To emulate the in vivo concentration that provided optimal CD206 ${ }^{+}$TAM targeting with minimal liver accumulation (30 $\mu \mathrm{M}$ in OG), we evaluated conjugates at $33 \mu \mathrm{M}$ DOX loading. Our previous studies comparing other mUNOtargeted vs. untargeted polymeric nanosystems ${ }^{44}$ demonstrated that the highest targeted uptake in primary M2 macrophages occurred after an interval of 10 to $30 \mathrm{~min}$. For this reason, we used an incubation time of $15 \mathrm{~min}$ for these experiments. Under these conditions, free DOX did not display toxicity to M2 or M1 macrophages (Fig. 3E, purple bar); in contrast, OximUNO displayed significantly higher toxicity against M2 macrophages than DOX (52\%) and St-PGA-DOX (33\%) (Fig. 3E, red bar) while OximUNO induced two times less toxicity than St-PGA-DOX in M1 macrophages. Interestingly, St-PGADOX showed higher toxicity in M1 than M2 macrophages (Fig. 3E, blue bar), possibly because M1 macrophages display greater phagocytic activity than M2 macrophages ${ }^{52,53}$, which might also explain the $60 \%$ cell viability following OximUNO treatment of M1 macrophages (Fig. $3 \mathrm{E}$, red bar). We observed a similar trend using a higher concentration of DOX $(100 \mu \mathrm{M})$; however, free DOX displayed significant toxicity in M2 macrophages at this higher concentration (Fig. 3E, purple bar). These results provide evidence that OximUNO displayed increased toxicity towards M2 macrophages when compared to St-PGA-DOX or DOX alone. We note that OximUNO induced a degree of in vitro toxicity towards M1 macrophages; however, our homing studies revealed that St-PGA-OG-mUNO did not target M1 macrophages

318 CD206 is also expressed on other macrophage populations in other organs, although at lower 319 levels than for M2 TAMs. We performed in vitro cytotoxicity studies of OximUNO on non320 polarised blood monocyte-derived macrophages, designated "M0", which we previously 321 showed express CD206, at levels lower than in M2 ${ }^{42}$. These assays revealed moderate toxicity 
of OximUNO to M0 macrophages (Fig. S13); however, the fact that St-PGA-OG-mUNO did

323 not target the lung, liver, or spleen (section 2) suggests that OximUNO will not affect the

324 macrophage populations of those organs.

325 We also evaluated the hepatic and renal safety profile of a single administration of OximUNO

326 (at doses corresponding to $2 \mathrm{mg} / \mathrm{kg}$ and $4 \mathrm{mg} / \mathrm{kg}$ DOX) by analysing creatinine (Crea) and

327 alanine aminotransferase (ALAT) levels $48 \mathrm{~h}$ after i.p. administration in healthy mice (Fig. 3F).

328 These doses did not induce toxic levels of Crea or ALAT compared to the values reported in the literature ${ }^{54}$ or the reference values for the female Balb/c reported in the Mouse Phenome Database by The Jackson Laboratory ${ }^{55}$ or Charles River facilities ${ }^{56}$. However, increased ALAT levels with the higher dose, prompted the selection of the OximUNO dose corresponding to 2 $\mathrm{mg} / \mathrm{kg}$ DOX for further in vivo studies.

A
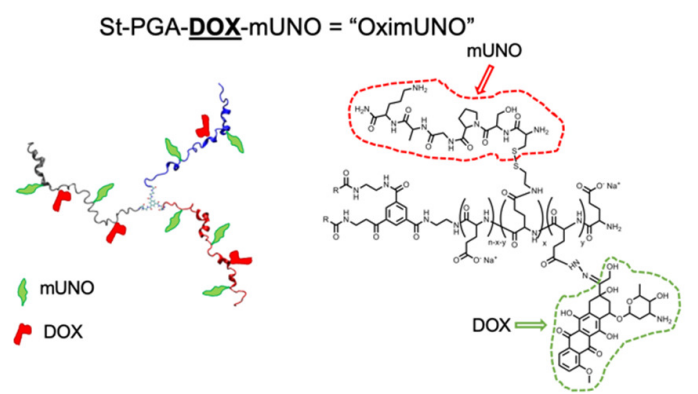

$\mathrm{C}$

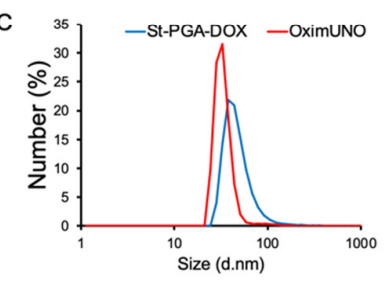

B

\begin{tabular}{ccccc} 
Compound & $\begin{array}{c}\% \text { wt } \\
\text { DOX }\end{array}$ & $\begin{array}{c}\% \text { wt } \\
\text { mUNO }\end{array}$ & $\begin{array}{c}\text { Size } \\
(\mathbf{d}, \mathbf{n m})\end{array}$ & $\begin{array}{c}\text { Zeta } \\
\text { potential } \\
(\mathbf{m V})\end{array}$ \\
\hline OximUNO & 10.77 & 3.56 & $37.48 \pm 7.44$ & $-41.0 \pm 5.07$ \\
\hline $\begin{array}{c}\text { St-PGA- } \\
\text { DOX }\end{array}$ & 8.08 & - & $\begin{array}{c}51.39 \pm \\
11.73\end{array}$ & $-49.8 \pm 3.97$ \\
\hline
\end{tabular}

D



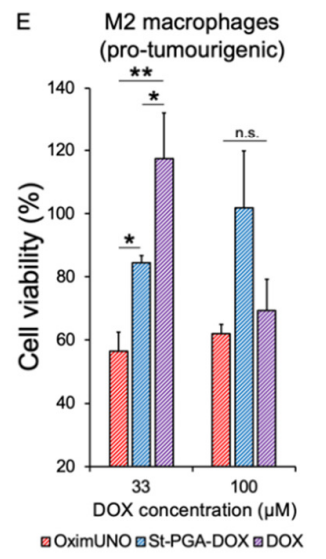

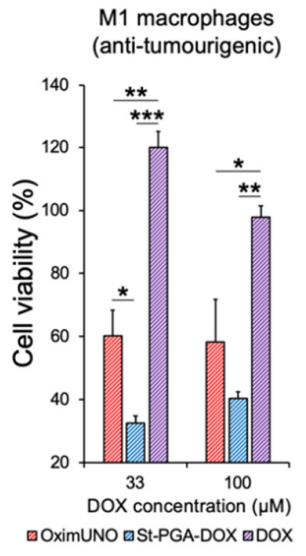

F

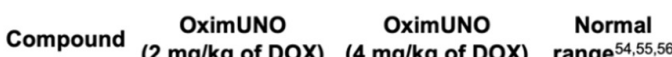

( $2 \mathrm{mg} / \mathrm{kg}$ of DOX) (4 mg/kg of DOX) range $54,55,56$

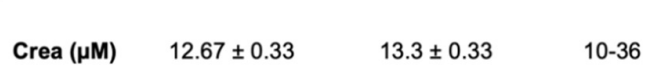

$\begin{array}{llll}\text { ALAT (U/I) } & 42 \pm 1.53 & 56 \pm 11.8 & 50-90\end{array}$

335 Figure 3. OximUNO enhances the in vitro efficacy of DOX on M2 macrophages. (A) Simplified form of

336 OximUNO (left) and molecular structure (right) showing St-PGA decorated with mUNO (red) and DOX (green). 
(B) Table showing DOX loading, mUNO loading, size (as measured by DLS), and charge (as measured by Zeta potential) of both nanoconjugates in PBS. (C) A DLS graph for measurements shown in (B), indicating the uniform size of OximUNO and St-PGA-DOX. (D) DOX release from OximUNO showing the drug release in PBS, i.p. fluid, acetate buffer or in the presence of cathepsin B. (E) In vitro cytotoxicity in primary human M2 (left panel) or M1 (right panel) macrophages after treatment with OximUNO (red bars), St-PGA-DOX (blue bars), and DOX (purple bars) following a 15 min incubation, washed, cultured for additional $48 \mathrm{~h}$, and then analysed for cell viability as evaluated by MTT assay. (F) Hepatic and renal toxicology serum levels of Crea and ALAT $48 \mathrm{~h}$ after i.p.-administration of OximUNO (at two different doses in DOX: $2 \mathrm{mg} / \mathrm{kg}$ and $4 \mathrm{mg} / \mathrm{kg}$ ) in healthy $\mathrm{Balb} / \mathrm{c}$ mice $(\mathrm{N}=3)$. Error bars represent the $\mathrm{SE}$ of the mean.

In summary, the conjugation of mUNO and DOX to the St-PGA backbone to yield OximUNO, enhanced the in vitro efficacy of DOX towards M2 macrophages with no in vivo renal or hepatic toxicity observed.

\section{OximUNO treatment of orthotopic TNBC depletes CD206 $^{+}$TAMs, inhibits $^{-}$}

\section{tumour progression and attenuates immunosuppression}

The findings of the in vivo homing and in vitro cytotoxicity studies supported the subsequent evaluation of OximUNO in the orthotopic TNBC model. When tumours reached $25 \mathrm{~mm}^{3}$, we treated mice with i.p. injections of OximUNO, St-PGA-DOX, or DOX, at $2 \mathrm{mg} / \mathrm{kg}$ DOX every other day for eighteen days. Encouragingly, OximUNO treatment significantly reduced primary tumour volume growth kinetics (Fig. 4A, red line) compared to DOX, St-PGA-DOX, and PBS. Furthermore, only the OximUNO treatment significantly reduced final tumour weight (Fig. 4B) compared to the untreated group. We assigned this encouraging therapeutic effect to mUNO-mediated targeting, as animals treated with the untargeted St-PGA-DOX possessed tumour volumes (Fig. 4A, blue line) similar to the PBS group (Fig. 4A, black line).

363 Furthermore, OximUNO treatment did not affect mouse bodyweight, whereas treatment with 364 DOX induced a significant decrease in mouse bodyweight starting from day twenty-one post365 inoculation (p.i.) until the end of the treatment (Fig. 4C).

366 Histological analysis of lungs from treated mice (Fig. S14 shows an H\&E stain from a healthy 367 lung for comparison) revealed that OximUNO also influenced the extent of pulmonary 368 metastases (Fig. 4D), as it elicited the highest reduction in the metastatic lung area and nodule 369 number ( $\mathrm{p}=0.06$ vs. PBS and $\mathrm{p}=0.06$ vs. DOX, respectively (Fig. 4E, F)). Meanwhile, 370 immunofluorescence (IF) microscopy revealed no significant changes in CD31 expression in 371 tumours (Fig. S15A, B), but significantly fewer CD31 ${ }^{+}$structures in the OximUNO-treated 
372 mice compared to DOX-treated mice (Fig. S15A, C), suggesting that the reduction in nodule 373 number in the OximUNO group (of Fig. 4F) may be mediated by the lower vascularisation in 374 the primary tumour. Importantly, histological analysis revealed no cardiotoxicity in any 375 treatment groups (Fig. S16). IF analysis revealed that only OximUNO significantly reduced 376 the CD206 expression (assigned to CD206 ${ }^{+}$TAMs), compared to PBS (Fig. 4G, J). 377 Interestingly, treatment with DOX upregulated CD206 expression (Fig. 4G, J), which agrees 378 with previous reports that demonstrated an increase in the number of CD206 ${ }^{+}$TAMs following 379 chemotherapy ${ }^{24}$.

380 Notably, only OximUNO treatment significantly increased CD8 expression (a marker of 381 cytotoxic T cells (CTLs)) compared to PBS and DOX treatment (Fig. 4H, K). Unexpectedly, 382 St-PGA-DOX treatment increased the expression of FOXP3, a marker for regulatory $\mathrm{T}$ cells 383 (Tregs) (Fig. 4I, L). Analysis of the CD8/FOXP3 expression ratio revealed that OximUNO 384 treatment resulted in a five-fold increase compared to St-PGA-DOX or DOX treatment (Fig. $3854 \mathrm{M})$, suggesting that OximUNO stimulated a shift in the immune landscape towards 386 immunostimulation. Of note, in all cases, we normalised the quantification of marker 387 expression using immunofluorescent images to the tissue area to account for different amounts 388 of tissue in different images. 

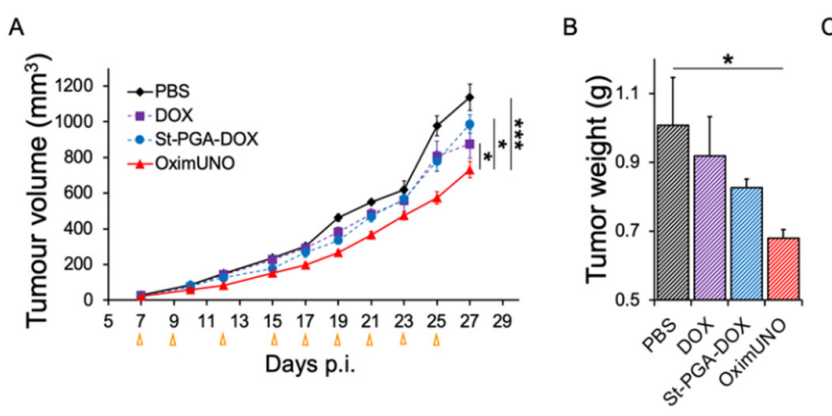

C
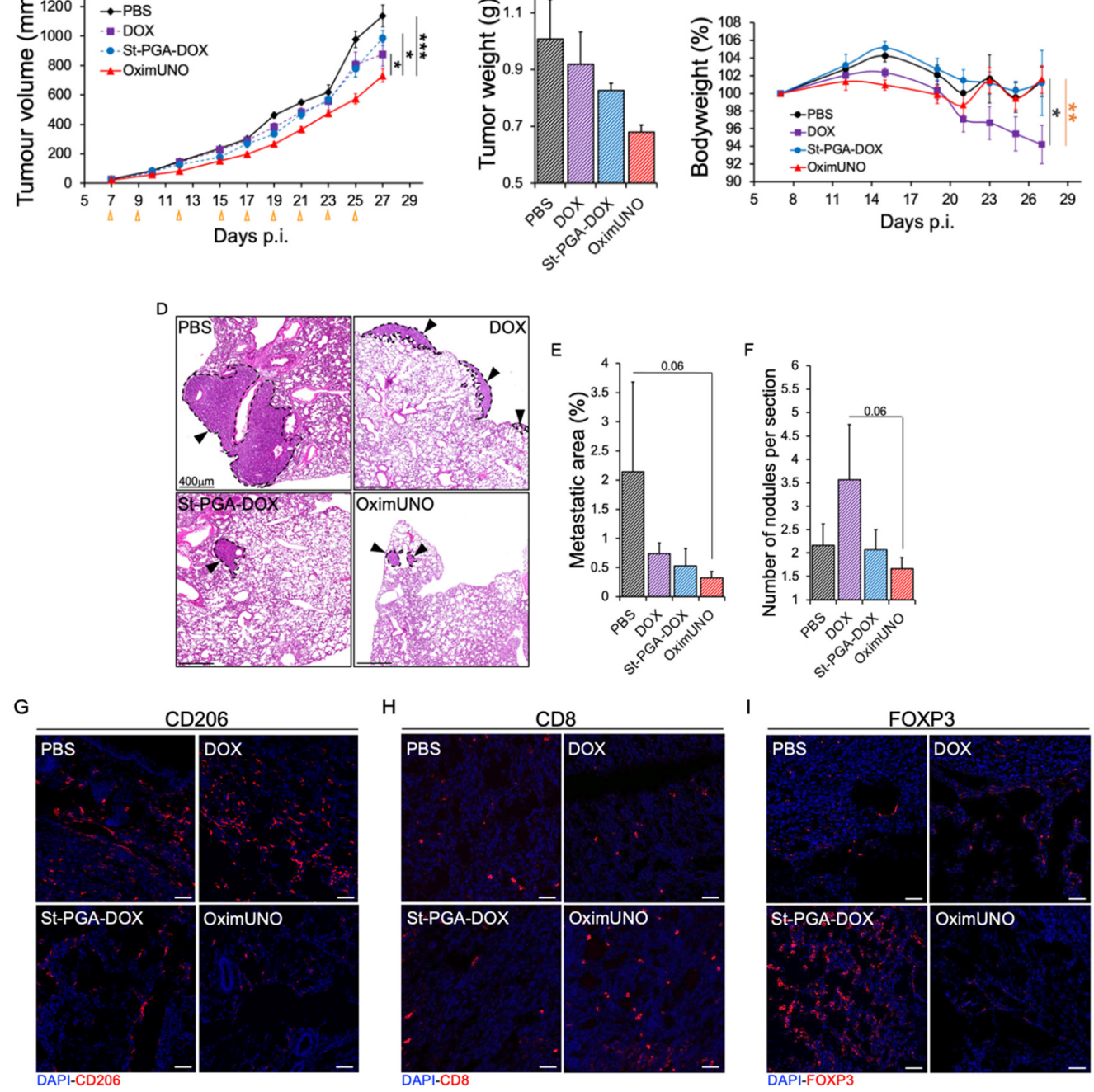

G
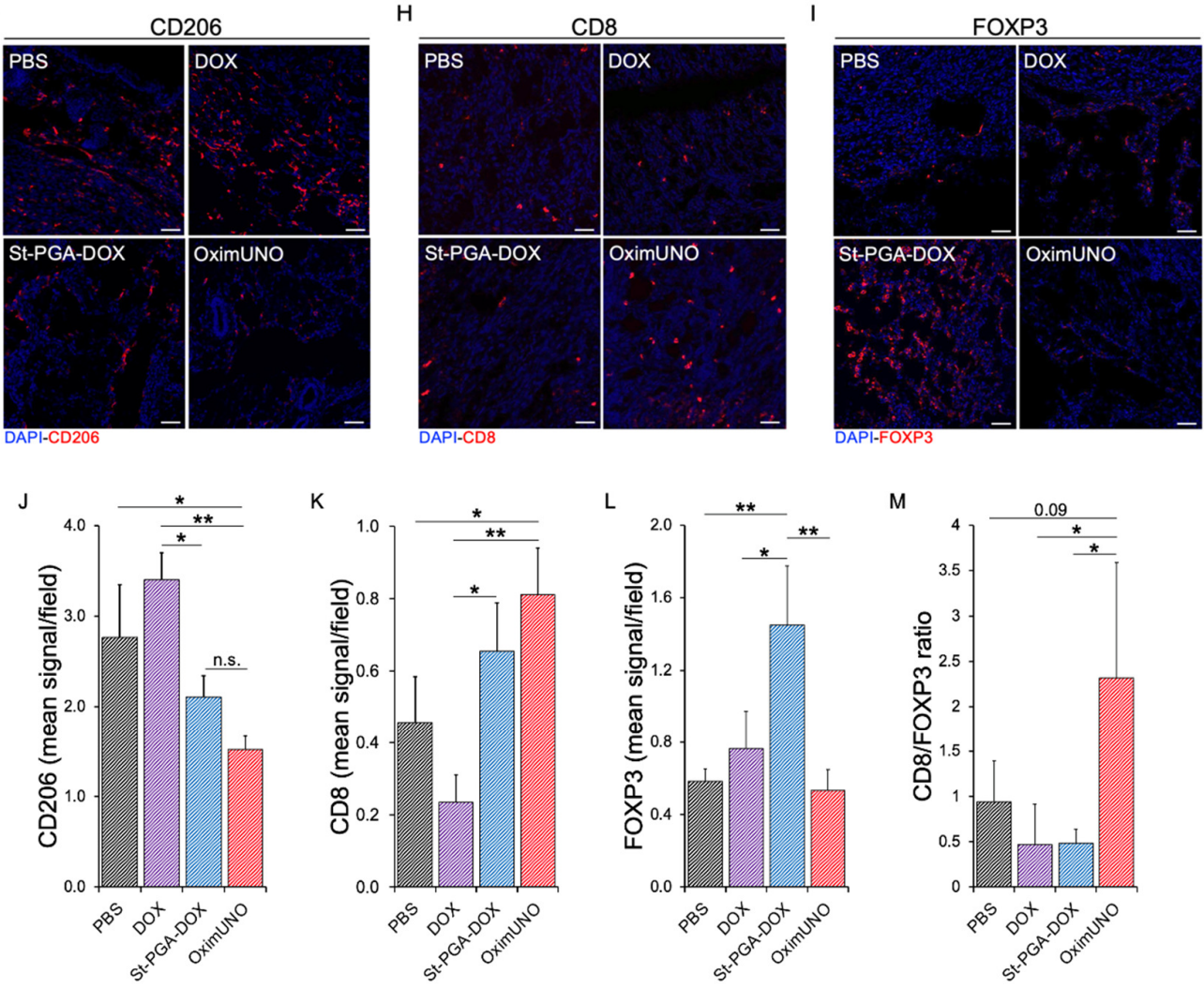

Figure 4. OximUNO treatment reduces primary tumour growth and pulmonary metastases and alleviates immunosuppression. Treatment with OximUNO, St-PGA-DOX, or DOX at $2 \mathrm{mg} / \mathrm{kg}$ DOX in mice bearing orthotopic TNBC tumours (N=5). I.p. injections began when tumours reached $25 \mathrm{~mm}^{3}$ and were performed every other day to give a total of nine injections. (A) Primary tumour volume progression during treatment. Orange 
arrows indicate injection days. (B) Primary tumour weight at the experimental endpoint, demonstrating a significantly smaller weight for OximUNO-treated mice (red bar) than other groups. (C) Mouse bodyweight analysis suggests the safety of OximUNO treatment (red line); meanwhile, DOX-treatment induced a significant reduction in bodyweight by the experimental endpoint (purple line). Dark grey $*$ DOX vs. PBS, orange $*$ DOX vs. St-PGA-DOX. (D) Representative H\&E images showing pulmonary metastases for all groups (scale bars = $400 \mu \mathrm{m})$; OximUNO treatment associated with the smallest metastatic area (E) and the lowest number of average nodules per lung (F). (G-I) Representative confocal microscopy images demonstrating the expression of (G) CD206, (H) CD8, and (I) FOXP3. Scale bars $=50 \mu \mathrm{m}$. (J-M) Quantification of confocal microscopy images for the expression of (J) CD206, (K) CD8, and (L) FOXP3. (M) Graph of CD8/FOXP3 expression ratio showing a shift in the immune profile. Quantification was performed using the ImageJ programme from at least three images per mouse and five mice per group. Error bars represent the SE of the mean.

By targeting CD206 ${ }^{+}$TAMs with DOX via OximUNO treatment, we increased the efficacy and reduced the toxicity of DOX in the orthotopic TNBC model. Our results also suggest that the depletion of CD206 ${ }^{+}$TAMs by OximUNO elicited an immunostimulatory shift.

OximUNO treatment of experimental TNBC metastasis reduces CD206 $^{+}$TAMs $^{-1}$ number, tumour burden and attenuates immunosuppression

We next evaluated the effect of OximUNO on experimental TNBC metastasis using GFPlabelled 4T1 cells. We treated mice every other day with i.p. injections of OximUNO, St-PGADOX, or DOX, starting from day four p.i. and sacrificed mice on day eighteen p.i. Analysis of whole lung fluorescence in the green channel revealed that OximUNO treatment induced the lowest GFP fluorescence, indicating a lower level of pulmonary metastases (Fig. 5A). Representative macroscopic images also provided evidence for a reduction in metastases (Fig. 5B). Confocal fluorescence microscopy of lungs confirmed the trend observed with whole lung fluorescence, showing fewer GFP fluorescent nodules in the OximUNO-treated group (Fig. 5C). Furthermore, histological analysis of lungs displayed the lowest number of pulmonary nodules for OximUNO-treated mice (Fig. 5D). Mice treated with the untargeted St-PGA-DOX and free DOX showed a significant decrease in bodyweight, resulting in a 19\% (Fig. 5E, blue line) and 17\% loss (Fig. 5E, purple line), respectively; meanwhile, OximUNO-treated mice displayed lower bodyweight loss (Fig. 5E, red line).

428 We next employed flow cytometry (FC) to analyse the effect of different treatments on the 429 immune cell populations in whole lungs. This analysis demonstrated that OximUNO treatment 430 significantly lowered the percentage of M2 TAMs (CD206 ${ }^{+}$(Fig. 5F) but did not significantly 
431 impact the percentage of M1 TAMs, CTLs, or Tregs (Fig. 5G-I). We observed the same trend 432 when we expressed these populations as total cell counts (Fig. S17-S20).

433 To evaluate if OximUNO affected CD206 ${ }^{+}$macrophages other than M2 TAMs, we analysed 434 the state of splenic macrophages from this treatment study using FC. This analysis revealed no 435 significant differences in the CD206/CD86 populations between the OximUNO-treated mice 436 and PBS-treated mice (Fig. S21A-C).

437 While FC analysis informs on the immune status of the whole lung, it does not provide specific 438 information regarding the TME. To characterise the immune landscape of the TME, we next 439 analysed the expression of markers for TAMs, CTLs, and Tregs in pulmonary nodules using 440 IF. This analysis revealed significantly lower CD206 expression in OximUNO-treated mice 441 than PBS-treated mice (Fig. 5J, N), providing evidence for a robust reduction in the number of $442 \mathrm{CD}^{206^{+}}$TAMs in the TME. Importantly, and similarly to OximUNO treatment in the 443 orthotopic TNBC mouse model, OximUNO elicited the highest expression of CD8 (Fig. 5K, 444 O). OximUNO and St-PGA-DOX treated mice demonstrated significantly lower lung FOXP3 445 expression than PBS- and DOX-treated mice (Fig. 5L, P). OximUNO-treated mice displayed 446 between a two- and three-times higher CD8/FOXP3 expression ratio than St-PGA-DOX and 447 DOX, and nearly seven-times higher than PBS (Fig. 5M). Therefore, our IF analysis in the 448 pulmonary tumour nodules suggested that OximUNO triggered a shift in the immune profile 449 of the TME towards immunostimulation. 
A
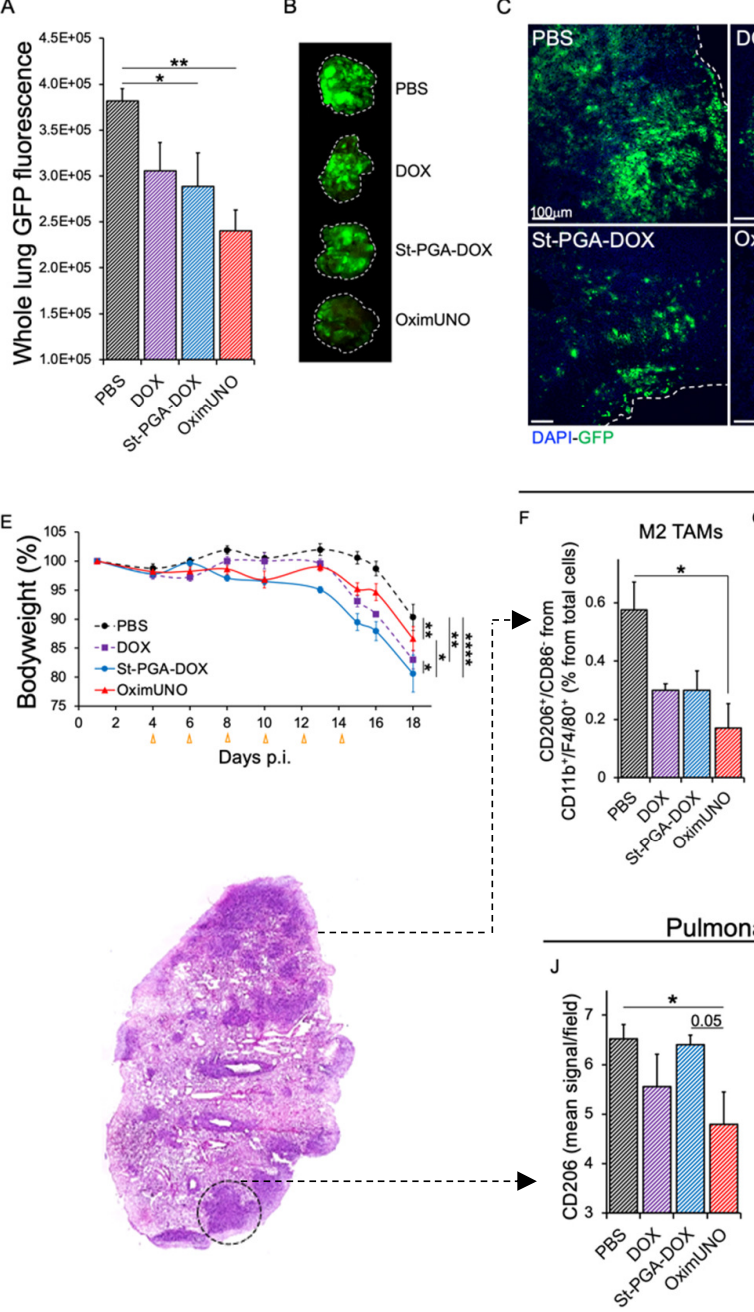

Pulmonar
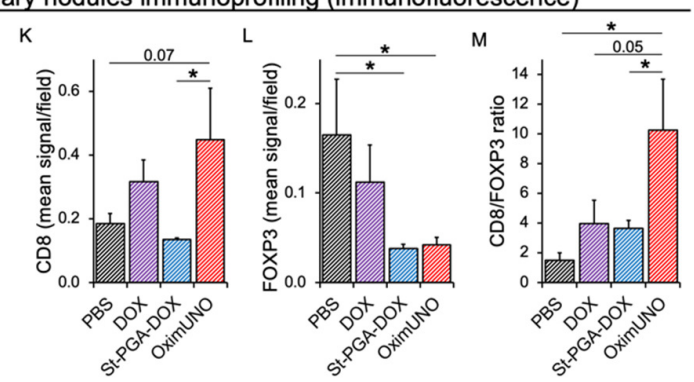

N

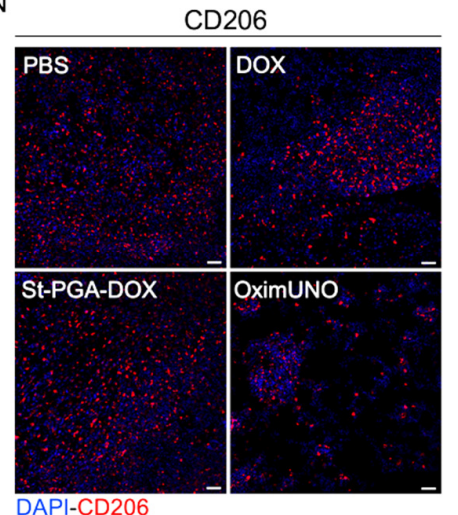

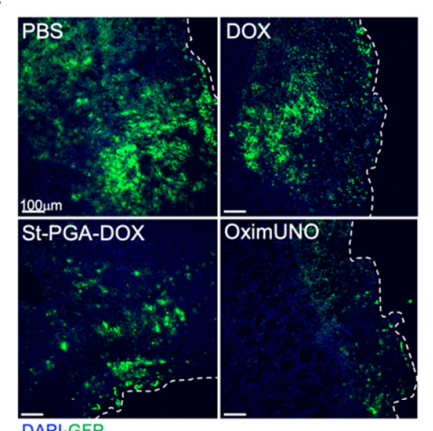

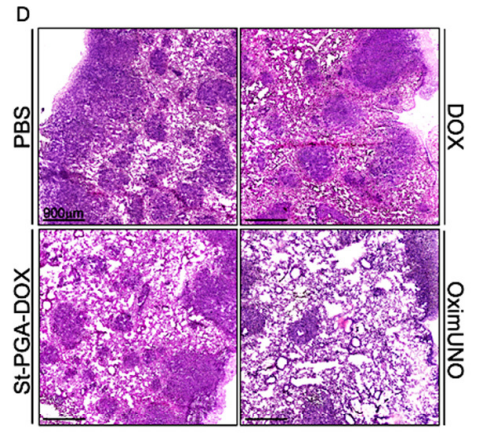

Whole lung immunoprofiling (cytometry)
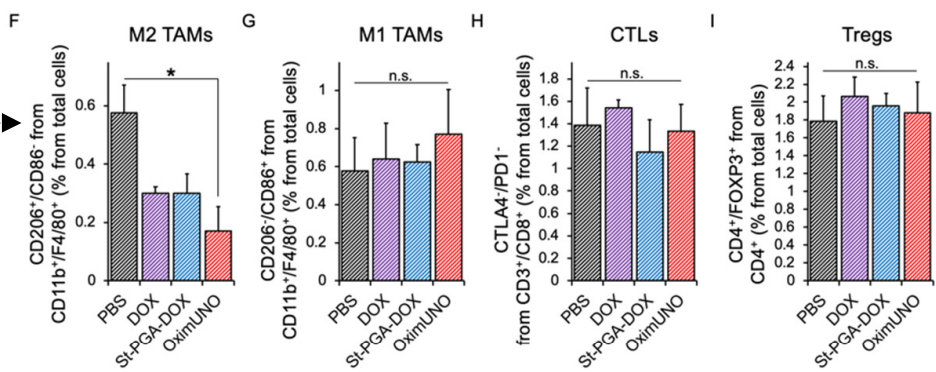

O

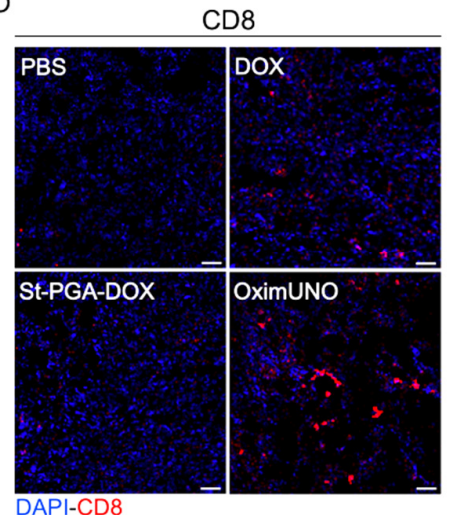

$\mathrm{P}$

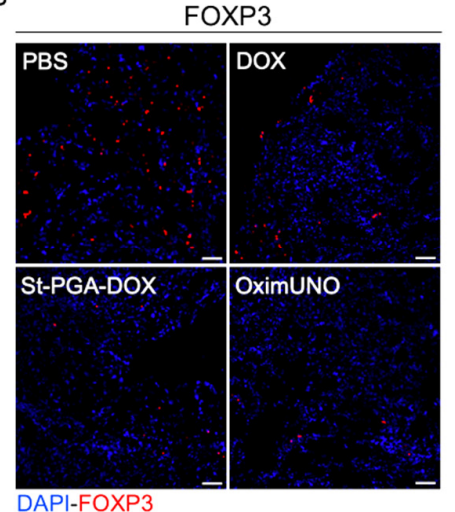
number and tumour burden and alleviates immunosuppression. Treatment with OximUNO, St-PGA-DOX, or DOX at $2 \mathrm{mg} / \mathrm{kg}$ DOX in the experimental metastasis of TNBC model, created using GFP-labelled 4T1 cells $(\mathrm{N}=6)$. I.p. injections began on day four p.i. and were performed every other day to give a total of six injections. 
(N=6). (B) Representative macroscopic photographs of GFP fluorescence in the lungs. (C) Representative confocal microscopy images of GFP expression, scale bars $=100 \mu \mathrm{m}$. (D) Representative H\&E images showing pulmonary metastases for all groups (scale bars $=900 \mu \mathrm{m}$ ). (E) Mouse bodyweight analysis, demonstrating significantly lower bodyweight lost with OximUNO (red line) compared with St-PGA-DOX-treated mice (blue line) and DOX-treated mice (purple dotted line). Orange arrows indicate injection days. (F-I) FC analysis on three right lungs per group. (F) M2 TAMs (CD206+), (G) M1 TAMs, (H) CTLs and (I) Tregs. (J-M) IF analysis on the pulmonary tumour nodules to detect the expression of (J) CD206, (K) CD8, and (L) FOXP3. (M) Graph showing CD8/FOXP3 expression ratio. IF images quantified using the ImageJ programme from at least five images per mouse and three mice per group. (N-P) Representative confocal microscopy images for (N) CD206, (O) CD8, and $(\mathbf{P})$ FOXP3. Scale bars $=50 \mu \mathrm{m}$. Error bars represent the SE of the mean.

By targeting DOX to CD206 ${ }^{+}$TAMs in experimental TNBC metastases, we increased the efficacy and reduced the toxicity of DOX, as OximUNO treatment associated with the presence of fewer pulmonary tumour lesions and less bodyweight loss when compared to treatment with untargeted St-PGA-DOX and DOX. Our results suggest that the observed therapeutic effect derived from $\mathrm{CD}_{206^{+}}$TAM depletion, which elicited an immunological shift in the TME.

\section{DISCUSSION}

To date, TNBC remains an aggressive breast cancer subtype ${ }^{3}$ with few treatment options, with conventional chemotherapy representing the current standard of care ${ }^{20}$. ICIs for TNBC have provided only modest improvements in complete response and progression-free survival in a small subset of TNBC patients ${ }^{9,12,15,16}$. Targeting TAMs can potentiate ICIs and other modalities and, therefore, represents an intense area of study ${ }^{57-61}$; however, TAMs represent a diverse population ${ }^{62-64}$, and which TAM subtype to target remains under investigation. Promising TAM-focused interventions under clinical evaluation include antibody-mediated depletion of TREM2-expressing TAMs (clinical trial identifier: NCT04691375). Antibody blockade of Clever-1 on M2 TAMs stimulated an M2 $\rightarrow$ M1 switch in TNBC models (4T1) and synergised with the PD-1 blockade ${ }^{67}$. Appealing studies have used anti-CD163 antibodies to target TAMs ${ }^{65}$ by decorating DOX-carrying liposomes with anti-CD163, to deplete TAMs and 498 potentiate ICIs in melanoma. Given our data comparing the tumour penetration of an anti499 PDL1 antibody vs. St-PGA-OG-mUNO, anti-CD163 systems may also display lower tumour 500 accumulation than St-PGA-OG-mUNO and OximUNO. Strategies targeting generic TAM markers such as CSF1R and CCR2 have shown side effects and limited efficacy. 
502 Motivated by the preponderance of the mannose receptor in tumourigenic/metastatic TAMs in 503 breast cancer ${ }^{68-70}$, here, we set out to deplete CD206 ${ }^{+}$TAMs in aggressive and metastatic 504 TNBC models and study the consequences on the progression and immunosuppressive state of 505 the tumour. To target CD206, a CD206-binding nanobody was developed by Ginderachter et 506 al. $^{71}$ which showed homing to CD206 ${ }^{+}$TAMs in in vivo models of lung and breast cancers ${ }^{71}$. 507 Navidea Inc. engineered a mannosylated compound ${ }^{72}$ (Manocept $\left.{ }^{\mathrm{TM}}\right)$, that forms part of the 508 FDA-approved contrast agent Lymphoseek®. Unfortunately, mannose-based ligands have 509 other binding partners besides CD206, including CD209 in intestinal and genital tissues ${ }^{45}$, and 510 can target dendritic cells ${ }^{46}$. Riptide Inc. also designed a peptide (RP-182) that binds to CD206; 511 however, the peptide also binds to RelB, Sirp- $\alpha$ and CD $47^{73}$.

512 We recently identified and described a short peptide called mUNO (sequence: CSPGAK) that 513 targets mouse ${ }^{41}$ and human CD206 ${ }^{43}$ at a different binding site than for mannose on CD206 ${ }^{43}$.

514 We identified mUNO from an in vivo screen using a peptide library in mice bearing metastatic 515 breast cancer; we subsequently described how mUNO homed to CD206 ${ }^{+}$TAMs in other solid 516 tumour models ${ }^{41,74}$ and in early-stage models of $\mathrm{TNBC}^{42}$ displaying low liver accumulation.

517 We envisioned that conjugating mUNO to St-PGA would significantly enhance targeting 518 through the avidity effect and increased plasma half-life ${ }^{75}$.

519 Compared to synthetic polymers such as N-(2-hydroxypropyl) methacrylamide, polypeptide520 based nanocarriers show several benefits, including biodegradability, lower immunogenicity, 521 and a lack of long term-accumulation, and the number of polypeptide-based constructs reaching 522 clinical evaluation has significantly increased in recent years ${ }^{76-78}$. We employed St-PGA-based 523 nanoconjugates with three linear chains ( $\sim 50$ glutamic acids each) linked to a central core. 524 Overall, the safety, lack of toxicity, and biodegradability of St-PGA meet FDA approval 525 criteria $^{79}$. A previous screen of PGA structures suggested that larger architectures enhanced 526 plasma half-life and increased bioavailability through a higher hydrodynamic volume that 527 reduces rapid renal clearance ${ }^{47,80}$. Of note, an extended plasma half-life will be advantageous 528 when targeting the continuously replenished TAM cell type ${ }^{81,82}$.

529 St-PGA-OG-mUNO, a fluorescent counterpart of OximUNO, can be easily monitored by 530 immunostaining for OG or detecting native OG fluorescence (as for the half-life study). Given 531 weak DOX fluorescence and the inability to detect DOX with an antibody, we first designed 532 St-PGA-OG-mUNO for validation purposes. We then exchanged OG for DOX to generate St533 PGA-DOX-mUNO, referred to as "OximUNO".

534 Our studies demonstrated that St-PGA-OG-mUNO displayed a far greater plasma half-life and 535 specificity to $\mathrm{CD}_{206^{+}} \mathrm{TAMs}$ than free mUNO and avoided CD86 ${ }^{+}$M1 TAMs and CD11c ${ }^{+}$ 
536 DCs, an important fact since M1 TAMs display anti-tumourigenic activity ${ }^{25}$, and CD11c ${ }^{+}$DCs 537 participate in antigen presentation ${ }^{83}$. In line with these observations, the computational analysis 538 indicated that mUNO peptides are available to a receptor and sweep a vast space $\left(130^{\circ}\right)$ around 539 PGA. Altogether this data demonstrates the benefit of conjugating mUNO to St-PGA. While 540 previous studies have reported the St-PGA nanocarrier ${ }^{47,79}$ and the mUNO targeting peptide ${ }^{42}$, 541 this work represents the first design of a peptide-targeted St-PGA nanosystem. Regarding the 542 administration route of peptide-guided St-PGA nanosystems, in the future we also wish to 543 evaluate the i.v. route, which, barring the case of intraperitoneal chemotherapy, represents a 544 more clinically translatable route to deliver cancer therapies.

545 In the OximUNO system, drug release studies revealed only 15\% DOX release, which agrees 546 with our previous studies ${ }^{48,50}$ but suggests room for improvement, which may come from using 547 longer polymer-drug linkers such as EMCH ( $\mathrm{N}$ - $\varepsilon$-maleimidocaproic acid hydrazide) moiety ${ }^{48,50}$ 548 or from the use of external triggers ${ }^{84-86}$. Unexpectedly, we failed to observe a significant 549 increase in DOX release in the presence of cathepsin B with respect to the hydrolytic 550 conditions; we hypothesise that the nanoconjugate conformation slows down proteolytic 551 degradation, hampering in vitro quantification within the studied timeframe ${ }^{47}$.

552 Our in vivo efficacy studies showed that, strikingly, the sole depletion of CD206 ${ }^{+}$TAMs with 553 OximUNO alleviated tumoural immunosuppression and reduced dissemination and growth, 554 confirming the pro-tumoural and immunosuppressive roles assigned to CD206 ${ }^{+}$TAMs in the 555 literature and reaffirming the importance of targeting this particular TAM subset. Additionally, 556 the observed reduction in the number of CD206 ${ }^{+}$TAMs and CD31 ${ }^{+}$structures for OximUNO 557 agrees with the established angiogenic role of $\mathrm{CD} 206^{+} \mathrm{TAMs}^{24}$.

558 From a safety point of view, we found that the OximUNO nano-formulation of DOX had the 559 least negative impact on mouse bodyweight compared to free DOX or the untargeted nano560 formulation St-PGA-DOX. Additionally, OximUNO did not alter Crea or ALAT levels, 561 indicating the absence of acute hepatic or renal toxicity. Our data suggest that the signal 562 observed in the kidneys for St-PGA-OG-mUNO (consistent with the previously reported 563 excretion of St-PGA ${ }^{47,79}$ ) did not translate into acute renal toxicity for OximUNO. These are 564 relevant findings as DOX induces cell death and tissue damage not only in the heart but also in 565 the liver and kidneys ${ }^{87}$. OximUNO displayed moderate toxicity to un-polarised macrophages 566 in vitro; however, OximUNO did not affect or alter the macrophage populations of the spleen 567 in vivo, in agreement with the absence of spleen targeting we observed for St-PGA-OG568 mUNO. 
569 Most preclinical studies evaluating the effect of M2 TAM targeted monotherapy in the 4T1 570 mouse model have either not shown efficacy on secondary tumours ${ }^{88,89}$, a lack of efficacy in 571 primary tumours or metastases in the case of anti-CLEVER-1 ${ }^{67}$, or a pro-metastatic effect in 572 the case of anti-CSF1R ${ }^{90}$. Hence, along with anti-MARCO therapy ${ }^{91}$, OximUNO constitutes 573 one of the few reports of an M2 TAM targeted monotherapy affecting both primary and 574 secondary tumours in the 4T1 mouse model.

575 Beyond TAM depletion, we show that St-PGA-mUNO represents an attractive platform to 576 carry additional therapeutic payloads other than DOX, which could include M2 $\rightarrow$ M1 577 polarising agents such as TLR7 agonists $^{44,92}$, beta-emitting radiotherapeutic agents such as 578 dodecanetetraacetic acid-chelated ${ }^{177} \mathrm{Lu}^{93}$, or photosensitisers used in photodynamic therapy ${ }^{84-}$

$579{ }^{86}$. We also envisage the combination of TAM-depletion via OximUNO administration together 580 with current chemotherapy regimens to prevent dissemination and relapse.

581 Taking OximUNO as a proof-of-concept, our data support the peptide-targeted St-PGA design 582 reported here as a new targeted nanosystem that could target other receptors by exchanging the 583 targeting peptide.

586 MATERIALS AND METHODS

\section{Reagents and solutions}

588 The peptides mUNO (sequence: CSPGAK-COOH) and FAM-mUNO (FAM-Ahx-CSPGAK$589 \mathrm{COOH}$ ) were purchased from TAG Copenhagen and doxorubicin (DOX) from Sigma-Aldrich. 590 St-PGA was kindly provided by Polypeptide Therapeutic Solution S.L. (PTS, Valencia, Spain). 591 See the Supplementary Information for information on all other reagents and solutions.

592 Mayer's haematoxylin solution was prepared by dissolving $5 \mathrm{~g}$ of aluminium potassium 593 sulphate dodecahydrate (Merck Millipore, cat. 1010421000) in $100 \mathrm{~mL}$ of water, and adding 1 $594 \mathrm{~g}$ of haematoxylin (Merck, cat. H9627). After complete dissolution, $0.02 \mathrm{~g}$ of sodium iodide 595 (Merck, cat. 1065230100) was added and completely dissolved. Then, $2 \mathrm{~mL}$ of acetic acid 596 (Sigma-Aldrich, cat. 33209) was added, and then the solution was boiled and then cooled. Once 597 ready to use, the solution was filtered using a $0.45 \mu \mathrm{m}$ filter.

598 Eosin (5\%) solution was prepared by dissolving $0.5 \mathrm{~g}$ of Eosin Y (Sigma-Aldrich, cat. 230251) 599 in $99 \mathrm{~mL}$ water/ $1 \mathrm{~mL}$ acetic acid.

600

601 Cell culture and experimental animals 
602 4T1 cells were purchased from ATCC, and 4T1-GFP cells were a gift from Ruoslahti

603 laboratory (Sanford-Burnham-Prebys Medical Discovery Institute, La Jolla, USA). 4T1 and

604 4T1-GFP cells were cultured in RPMI-1640 medium (Gibco by Life Technologies, cat. 72400-

605 021) supplemented with 10\% v/v foetal bovine serum (FBS, Capricorn Scientific, cat. FBS-

606 11A) and $100 \mathrm{IU} / \mathrm{mL}$ penicillin/streptomycin (Capricorn Scientific, cat. PS-B) at $37{ }^{\circ} \mathrm{C}$ in the

607 presence of $5 \% \mathrm{CO}_{2}$. For all animal experiments, 8-12-week-old female Balb/c mice were used.

608 Animal experiment protocols were approved by the Estonian Ministry of Agriculture (Project

609 \#159). All methods were performed in accordance with existing guidelines and regulations.

611 Tumour models

612 Two tumour models were used for homing studies: the orthotopic TNBC model, where $1 \times 10^{6}$

$6134 \mathrm{~T} 1$ cells in $50 \mu \mathrm{L}$ of phosphate-buffered saline (PBS, Lonza, cat. 17-512F) were

614 subcutaneously (s.c.) injected into the fourth mammary fat pad, and the experimental metastasis

615 of TNBC model, where $5 \times 10^{5} 4 \mathrm{~T} 1$ cells in $100 \mu \mathrm{L}$ of PBS were injected i.v. into Balb/c mice.

616 Two tumour models were used for treatment studies: the orthotopic TNBC model where $5 \times 10^{4}$

$6174 \mathrm{~T} 1$ cells in $50 \mu \mathrm{L}$ of PBS were injected s.c. into fourth mammary fat pad; and the experimental

618 metastasis of TNBC model where $2 \times 10^{5}$ 4T1-GFP cells in $100 \mu \mathrm{L}$ of PBS were i.v. injected.

620 Nanoconjugate synthesis and characterisation

621 In vivo homing studies used St-PGA-OG and St-PGA-OG-mUNO, while in vitro cytotoxicity

622 and in vivo treatment studies used St-PGA-DOX and St-PGA-DOX-mUNO ("OximUNO").

623 DOX denotes doxorubicin. Detailed synthetic procedures for single nanoconjugates can be

624 found in Supplementary Information.

625

626 Physico-chemical characterisation methods

627 Nuclear magnetic resonance (NMR) spectroscopy: NMR spectra were recorded at $27{ }^{\circ} \mathrm{C}(300$

$628 \mathrm{~K})$ on a 300 UltrashieldTM from Bruker. Data were processed with Mestrenova software.

629 Sample solutions were prepared at the desired concentration in $\mathrm{D}_{2} \mathrm{O}$ or $\mathrm{D}_{2} \mathrm{O}$ supplemented with

$630 \mathrm{NaHCO}_{3}(0.5 \mathrm{M})$.

631 UV-Visible (UV-Vis) analysis: UV-Vis measurements were performed using JASCO V-630

632 spectrophotometer at $25^{\circ} \mathrm{C}$ with $1 \mathrm{~cm}$ quartz cells and a spectral bandwidth of $0.5 \mathrm{~nm}$. Spectra

633 analysis was recorded three times in the range of 200-700 nm. 
634 Fluorescence analysis: Fluorescence analysis was performed using a JASCO FP-6500 635 spectrofluorimeter at $25^{\circ} \mathrm{C}$ with $1 \mathrm{~cm}$ quartz cells.

636 Dynamic Light Scattering (DLS): Size measurements were performed using a Malvern 637 ZetasizerNano ZS instrument, supported by a $532 \mathrm{~nm}$ laser at a fixed scattering angle of $173^{\circ}$.

638 Nanoconjugate solutions $(0.1 \mathrm{mg} / \mathrm{mL})$ were freshly prepared in PBS (10 mM phosphate, 150 $639 \mathrm{mM} \mathrm{NaCl}$ ), filtered through a $0.45 \mu \mathrm{m}$ cellulose membrane filter, and measured. Size 640 distribution was measured (diameter, $\mathrm{nm}$ ) for each polymer in triplicate. Automatic 641 optimisation of beam focusing and attenuation was applied for each sample.

642 Zeta potential measurements: Zeta potential measurements were performed at $25^{\circ} \mathrm{C}$ using a 643 Malvern ZetasizerNano ZS instrument, equipped with a $532 \mathrm{~nm}$ laser using disposable folded 644 capillary cells, provided by Malvern Instruments Ltd. Nanoconjugate solutions $(0.1 \mathrm{mg} / \mathrm{mL})$ 645 were freshly prepared in $1 \mathrm{mM} \mathrm{KCl}$. Solutions were filtered through a $0.45 \mu \mathrm{m}$ cellulose 646 membrane filter. Zeta potential was measured for each sample per triplicate.

\section{Molecular dynamics simulations}

649 Molecular dynamics (MD) simulations of PGA chains, and mUNO peptide were carried out 650 using the ff19SB force field ${ }^{95}$ in the Amber20 MD engine ${ }^{96}$. The nanoconjugate system was 651 neutralised using $\mathrm{Na}+$ ions and hydrated to account for a total of $\sim 920,000$ atoms $(\sim 300,000$ 652 TIP3P water molecules) in a truncated octahedral box. A hydrogen mass repartitioning strategy 653 was applied on the resulting topology, allowing us a 4 fs integration time step ${ }^{97}$. Standard 654 minimisation and equilibration protocols were used to reach $300 \mathrm{~K}$ and $1 \mathrm{~atm}$., followed by 50 655 ns of production MD run. The simulations were run under the NVT ensemble (constant number 656 of particles, volume, and temperature through Berendsen thermostat ${ }^{98}$ ), considering periodic 657 boundary conditions. The SHAKE algorithm was used to fix hydrogen atoms ${ }^{99}$. The non-bound 658 cut-off value was set to Angstrom. The central moiety was parametrised using the 659 recommended protocol for the Amber force field. It was necessary to introduce amide bond, 660 angle and dihedral terms using the ParmEd module to establish the bond of the central molecule 661 to the PGA chains.

662

\section{Tumour homing studies}

664 Tumours were induced as described in the tumour model section. Tumour homing studies were 665 performed on mice bearing orthotopic TNBC or experimental metastasis of TNBC. Ten days 666 p.i. of the orthotopic TNBC or the experimental metastasis of TNBC model, mice were i.p. 667 injected with St-PGA-OG-mUNO $(0.41 \mathrm{mg} / 0.5 \mathrm{~mL}$ of PBS) or St-PGA-OG $(0.35 \mathrm{mg} / 0.5 \mathrm{~mL}$ 
of PBS) (corresponding to 15 nmoles of OG, fluorescence measured by UV-Vis). The homing of a higher dose of St-PGA-PGA-mUNO $(0.82 \mathrm{mg} / 0.5 \mathrm{~mL}$ of PBS) or St-PGA-OG $(0.7$ $\mathrm{mg} / 0.5 \mathrm{~mL}$ of PBS) (corresponding to 30nmoles of OG) was also analysed and compared with the homing of FAM-mUNO (30nmoles/0.5mL of PBS). In every case, nanoconjugates or free peptide were circulated for $6 \mathrm{~h}$, after which mice were sacrificed by anaesthetic overdose followed by cervical dislocation. Organs and tumours were collected and fixed in cold $4 \% \mathrm{w} / \mathrm{v}$ paraformaldehyde (PFA) in PBS at $+4{ }^{\circ} \mathrm{C}$ for $24 \mathrm{~h}$, washed in PBS at room temperature for 1 $\mathrm{h}$ and cryoprotected in 15\% w/v sucrose (Sigma Life Science, cat. S9378) followed by 30\% w/v sucrose at $4{ }^{\circ} \mathrm{C}$ overnight. Cryoprotected and fixed tissues were frozen in OCT (Optimal Cutting Temperature, Leica, cat. 14020108926), cryosectioned at $10 \mu \mathrm{m}$ on Superfrost+ slides (Thermo Fisher, cat. J1800AMNZ) and stored at $-20{ }^{\circ} \mathrm{C}$. Immunofluorescent staining was performed as described earlier ${ }^{42}$. OG was detected using rabbit anti-FITC/Oregon Green (dilution 1/100, Invitrogen by Thermo Fisher Scientific, cat. A889) and Alexa Fluor 647 goat anti-rabbit antibody (dilution 1/250, Invitrogen by Thermo Fisher Scientific, cat. A21245). CD206 was detected using rat anti-mouse CD206 (dilution 1/150, Bio-Rad, cat. MCA2235GA) and Alexa Fluor ${ }^{\circledR} 546$ goat anti-rat antibody (dilution 1/250, life technologies, cat. A11081). CD86 was detected using rat anti-mouse CD86 (dilution 1/100, BioLegend, cat. 105001) and Alexa Fluor ${ }^{\circledR} 546$ goat anti-rat secondary antibody (dilution 1/250). CD11c was detected using hamster anti-mouse CD11c antibody (dilution 1/75, BioLegend, cat. 117301) and Alexa Fluor ${ }^{\circledR} 546$ goat anti-hamster secondary antibody (dilution 1/200, life technologies, cat. A21111) Slides were counterstained using 4',6-diamidino-2-phenylindole (DAPI, $1 \mu \mathrm{g} / \mathrm{mL}$ in PBS, Sigma-Aldrich, cat. D9542-5MG). Coverslips were mounted using mounting medium (Fluoromount-G ${ }^{\mathrm{TM}}$ Electron Microscopy Sciences, cat. 17984-25), and sections were imaged using Zeiss confocal microscope (Zeiss LSM-710) and 20x objective. The colocalisation analysis shown in Fig. 2M-P, between the FAM or OG channel and the CD206 channel was carried out using the "Coloc2" plugin in the Fiji programme and selecting the "Pearson's R value (no threshold)" coefficient. The colocalisation values were obtained from at least three representative images per mouse per group and their average and standard error were plotted. The OG/FAM mean signal per $\mathrm{CD}_{206^{+}}$cell analysis was measured using the ImageJ programme, taking the mean OG/FAM signal, and dividing it with the number of CD206 ${ }^{+}$cells. Average values were obtained from four images per mouse. $\mathrm{N}=3$ for orthotopic TNBC and $\mathrm{N}=2$ for the homing in experimental metastasis of TNBC. 
701 Analysis of tumour and liver leakiness

702 Endogenous IgG immunostaining of orthotopic 4T1 tumours and livers was performed 703 following the same IF protocol as described above to assess leakiness. Endogenous IgG was 704 detected using Alexa Fluor ${ }^{\circledR} 647$ goat anti-mouse antibody (dilution 1/200, Invitrogen by 705 Thermo Fisher Scientific, cat. A21235) and slides were counterstained with DAPI $(1 \mu \mathrm{g} / \mathrm{mL}$ in 706 PBS). The coverslips were mounted, and sections were imaged using Zeiss confocal 707 microscope and 20x objective ( $\mathrm{N}=3$ tumours).

708

\section{PDL1 expression analysis in orthotopic TNBC tumours}

710 The assessment of PDL1 expression in orthotopic 4T1 tumours followed the IF protocol 711 described above. PDL1 was detected using rat anti-mouse PDL1 (dilution 1/100, BioLegend, 712 cat. 124302) as primary antibody and Alexa Fluor® 647 goat anti-rat (dilution 1/200, 713 Invitrogen, cat. A21247) as the secondary antibody. Slides were counterstained with DAPI (1 $714 \mu \mathrm{g} / \mathrm{mL}$ in PBS), mounted, and imaged using a Zeiss confocal microscope.

\section{Tumour homing of anti-PDL1 in orthotopic TNBC tumours}

717 For the homing analysis with anti-PDL1, we injected $1 \times 10^{6} 4 \mathrm{~T} 1$ cells in $50 \mu \mathrm{L}$ of PBS s.c. and 718 ten days p.i., PDL1 antibody $(5 \mathrm{mg} / \mathrm{kg}$, rat anti-mouse, BioXcell, cat. BE0101) was injected 719 i.v., circulated for $24 \mathrm{~h}$ after which mice were sacrificed, organs collected and fixed with PFA. 720 Ten $\mu \mathrm{m}$ tissue sections were stained with Alexa Fluor® 647 goat anti-rat antibody (dilution 721 1/200), counterstained with DAPI (1 $\mu \mathrm{g} / \mathrm{mL}$ in PBS), mounted, and imaged with a Zeiss 722 confocal microscope.

\section{Plasma half-life evaluation for St-PGA-OG-mUNO}

725 Plasma half-life studies were performed as previously described ${ }^{42}$. Briefly, healthy female 726 Balb/c mice $(\mathrm{N}=3)$ were i.p. injected with St-PGA-OG-mUNO $(0.41 \mathrm{mg} / 0.5 \mathrm{~mL}$ of PBS, 727 corresponding to $15 \mathrm{nmoles} \mathrm{OG})$. Ten $\mu \mathrm{L}$ of blood was sampled at different timepoints $(0,5$, $72810,15,30,60,180,360$, and $1440 \mathrm{~min}$ ) and mixed with $50 \mu \mathrm{L}$ of PBS-Heparin solution. Blood 729 samples were centrifuged to obtain plasma (300g for $5 \mathrm{~min}$ at room temperature) and OG

730 fluorescence was read with a plate reader (FlexStation II Molecular Devices) at 480nm 731 excitation/520nm emission. 
734 LC-MS was implemented to determine free drug levels, stability, and drug release with

735 OximUNO. The LC-MS system consisted of an ExionLC LC system and AB Sciex QTRAP 7364500 , a triple quadrupole ion trap hybrid equipped with a Turbo VTM electrospray ionisation 737 source. DOX was detected with an internal standard method: $1 \mu \mathrm{g} / \mathrm{mL}$ of daunorubicin (DAU) 738 was used as internal standard, where three calibration curves (in a range from 0.5 to $50 \mu \mathrm{g} / \mathrm{mL}$ 739 DOX) were prepared and used for accurate analysis of DOX in the samples. Both DOX and 740 DAU were detected with positive electrospray ionisation mode by following two mass 741 transitions $(544.2 \mathrm{~m} / \mathrm{z} \rightarrow 397 \mathrm{~m} / \mathrm{z}$ and $544.2 \mathrm{~m} / \mathrm{z} \rightarrow 379 \mathrm{~m} / \mathrm{z}$ for DOX, and $528 \mathrm{~m} / \mathrm{z} \rightarrow 363.1$

$742 \mathrm{~m} / \mathrm{z}$ and $528 \mathrm{~m} / \mathrm{z} \rightarrow 321.3 \mathrm{~m} / \mathrm{z}$ for DAU). The obtained LC-MS optimal conditions were as 743 follows: flow rate $0.5 \mathrm{~mL} / \mathrm{min}$; mobile phase $-0.05 \%$ trifluoroacetic acid with $70 \%$ of 744 acetonitrile; LiChrospher $100 \mathrm{C} 18$ column (125x4.0 mm) (Merck); column temperature $40{ }^{\circ} \mathrm{C}$, $74510 \mu \mathrm{L}$ injection volume.

746 Stability study of OximUNO conjugate in PBS, pH 7.4

747 OximUNO was incubated in $10 \mathrm{mM}$ dPBS at $37^{\circ} \mathrm{C}$ at the concentration of $3 \mathrm{mg} / \mathrm{mL}$ and with $7483 \mu \mathrm{g} / \mathrm{mL}$ of DAU. $100 \mu \mathrm{L}$ aliquots were collected at defined time points $(0,1,2,5,24,48,72$ $749 \mathrm{~h}$ ), extracted with $3 \times 250 \mu \mathrm{L}$ chloroform, and mixed by vortexing for $5 \mathrm{~min}$. Organic phases 750 from all three chloroform extracts were collected in one tube, evaporated using speed vacuum, 751 and stored at $-20{ }^{\circ} \mathrm{C}$. On the day of analysis, dried samples were reconstituted in $300 \mu \mathrm{L}$ of 752 methanol (LC-MS grade), vortexed for $5 \mathrm{~min}$ and centrifuged for $5 \mathrm{~min}$ at 30,437g.

753 Supernatants were filtered through a $0.45 \mu \mathrm{m}$ filter and subjected to LC-MS analysis.

754 Stability study of OximUNO in the i.p. fluid

755 I.p. fluid was collected from healthy 8-12-week old Balb/c female mice as performed in $\mathrm{REF}^{100}$ 756 by collecting the supernatant and discarding the pellet after the centrifugation step. A working 757 solution containing $3 \mathrm{mg} / \mathrm{mL}$ of OximUNO and $3 \mu \mathrm{g} / \mathrm{mL}$ of DAU in i.p. fluid was incubated 758 at $37^{\circ} \mathrm{C} .50 \mu \mathrm{L}$ aliquots were collected at scheduled time points $(0,2,5,7$, and $24 \mathrm{~h})$. Samples 759 were then diluted with $100 \mu \mathrm{L}$ of methanol, sonicated to dissolve DOX, and injected into the 760 LC-MS after filtration through a $0.45 \mu \mathrm{m}$ filter.

761

762 Cathepsin B release kinetic studies.

763 Cathepsin B (5 IU) was activated in $2 \mathrm{mM}$ EDTA, $5 \mathrm{mM}$ DTT, and $20 \mathrm{mM} \mathrm{CH}_{3} \mathrm{COONa}$ buffer 764 and incubated at $37^{\circ} \mathrm{C}$ for $15 \mathrm{~min}$. In a separate tube, a solution containing $3 \mathrm{mg} / \mathrm{mL}$ OximUNO 765 and $3 \mu \mathrm{g} / \mathrm{mL}$ of DAU was prepared with $20 \mathrm{mM} \mathrm{CH}_{3} \mathrm{COONa}$ and incubated at $37{ }^{\circ} \mathrm{C}$ for 15 $766 \mathrm{~min}$. The two solutions were then combined to produce a reaction solution that was incubated 
at $37^{\circ} \mathrm{C} .100 \mu \mathrm{L}$ aliquots were collected at scheduled time points $(0,1,2,5,8,24,48,72 \mathrm{~h})$,

768 and after the addition of $900 \mu \mathrm{L}$ of dPBS (to adjust the $\mathrm{pH}$ level to 7.4), free DOX and DAU were extracted with $2.5 \mathrm{~mL}$ of $\mathrm{CHCl}_{3}$ three times. Samples were processed as described under "Stability Study of OximUNO conjugate in PBS, $\mathrm{pH}$ 7.4". After $\mathrm{CHCl}_{3}$ evaporation, samples were reconstituted with $300 \mu \mathrm{L}$ of methanol, filtered through a $0.45 \mu \mathrm{m}$ filter and subjected to LC-MS analysis. A blank solution was prepared with the same components as the sample solution but without cathepsin B and used as a control sample.

\section{In vitro cytotoxicity assay}

Human peripheral blood mononuclear cells (PBMC) were purified from human blood buffy coat using Ficoll Paque Plus (GE Healthcare, cat. 17-1440-02) reagent and CD14 ${ }^{+}$microbeads (MACS Miltenyi Biotec, cat. 130-050-201) as previously described ${ }^{42} .1 .2 \times 10^{5}$ cells in $50 \mu \mathrm{L}$ of RPMI-1640 medium were seeded on an FBS-coated 96-well plate. To obtain M0 macrophages, $50 \mu \mathrm{L}$ of macrophage colony stimulating factor (M-CSF) $(100 \mathrm{ng} / \mathrm{mL}$, BioLegend, cat. 574802) was added and replenished every other day for four days by substituting the half of the medium with fresh medium containing M-CSF. To obtain optimal macrophage attachment and M2 polarisation, $50 \mu \mathrm{L}$ of interleukin-4 (IL-4, $50 \mathrm{ng} / \mathrm{mL}$, BioLegend, cat. 574002) and M-CSF (100 ng/mL) mixture was added to the wells. The medium was replenished by substituting half of the medium with fresh medium containing IL-4 and MCSF every other day for six days. To obtain M1 macrophages, monocytes were incubated with M-CSF (100 ng/mL) for six days, replenishing every other day with fresh medium containing M-CSF and on day six, $50 \mu \mathrm{L}$ of M-CSF, lipopolysaccharide (LPS, $100 \mathrm{ng} / \mathrm{mL}$, Sigma Aldrich, cat. L4391) and interferon- $\gamma$ (IFN- $\gamma, 20 \mathrm{ng} / \mathrm{mL}$, BioLegend, cat. 570202) was added and incubated overnight. On day seven for M2 and M1 macrophages or day four for M0 macrophages, cells were incubated for $15 \mathrm{~min}$ at $37{ }^{\circ} \mathrm{C}$ with OximUNO, St-PGA-DOX, DOX in medium, or free medium as a control ( $\mathrm{N}=3$ wells per group). Concentrations used were calculated based on DOX: $33 \mu \mathrm{M}$ and 100 $\mu \mathrm{M}$. (Of note, the dose of OximUNO used for the 33 $\mu \mathrm{M}$ DOX experiments shown in Figure 3E corresponds to the same dose of OximUNO used for both in vivo treatment studies. In vivo, all treated groups received injections containing 2 $\mathrm{mg} / \mathrm{Kg}$ DOX, which, assuming the dilution in mouse blood, corresponds to a DOX concentration of $33 \mu \mathrm{M})$. After incubation, wells were washed, fresh medium added, and cells incubated for $48 \mathrm{~h}$ at $37{ }^{\circ} \mathrm{C}$. After $48 \mathrm{~h}, 10 \mu \mathrm{L}$ of 3-(4,5-dimethylthiazol-2-yl)-2,5diphenyltetrazolium bromide (MTT, concentration $5 \mathrm{mg} / \mathrm{mL}$, Invitrogen, cat. M6494) in PBS 
800

801

802

803

804

805

806

807

808

809

810

811

812

813

814

815

816

817

818

819

820

821

822

823

824

825

826

827

828

829

830

831

832

was added to each well containing culture medium and incubated for $2.5 \mathrm{~h}$ at $37^{\circ} \mathrm{C}$. Medium containing MTT was then removed without removing formed crystals, and $100 \mu \mathrm{L}$ of isopropanol was added to each well to dissolve crystals. Absorbance was read at $580 \mathrm{~nm}$ using a plate reader (Tecan Sunrise) and the corresponding Magellan ${ }^{\mathrm{TM}} 7$ programme.

\section{In vivo liver and kidney toxicology studies with OximUNO}

Three healthy 12-week-old female Balb/c mice were i.p. injected once with OximUNO (0.704 $\mathrm{mg} / 0.5 \mathrm{~mL}$ PBS or $1.408 \mathrm{mg} / 0.5 \mathrm{~mL}$ ) and circulated for $48 \mathrm{~h}$. Then, mice were anesthetised, and blood collected through retro-orbital bleeding into Lithium Heparin tubes (BD Vacutainer, cat. 368494). Blood samples were centrifuged at $1800 \mathrm{~g}$ for $15 \mathrm{~min}$ at $+4{ }^{\circ} \mathrm{C}$ and $400 \mu \mathrm{L}$ of plasma was collected for analysis. Samples were analysed in Tartu University Hospital using a Cobas 6000 IT-MW (Roche Diagnostics Gmbh) machine and reagents for creatinine (CREP2, cat. 03263991) and alanine aminotransferase (ALTLP, cat. 04467388).

\section{OximUNO treatment of orthotopic TNBC}

$5 \times 10^{4} 4 \mathrm{~T} 1$ cells in $50 \mu \mathrm{L}$ of PBS were s.c. injected into the fourth mammary fat pad of 8-12week-old female Balb/c mice. On day seven, mice were sorted into four groups by tumour volume measured using a digital calliper (Mitutoyo). Tumour volume was calculated based on the formula $\left(\mathrm{W}^{2} \mathrm{x} \mathrm{L}\right) / 2$, where $\mathrm{W}$ is the tumour's width and $\mathrm{L}$ is the tumour's length. The starting volume for each group was $\sim 25 \mathrm{~mm}^{3}$, and the number of mice in each group was five. The first i.p. injection of compounds was carried out on day seven, followed by an i.p. injection every other day; nine injections were performed in total. The dose of nanoconjugates was calculated based on DOX, 2mg/kg per injection (DOX: $39.5 \mu \mathrm{g} / 0.5 \mathrm{~mL}$ PBS; St-PGA-DOX: $476 \mu \mathrm{g} / 0.5 \mathrm{~mL}$ PBS; OximUNO: $341 \mu \mathrm{g} / 0.5 \mathrm{~mL}$ PBS) giving a cumulative dose of DOX of 18 $\mathrm{mg} / \mathrm{kg}$. Mouse bodyweight and tumour volumes were monitored every other day. The final injection was on day 25 and all mice were sacrificed on day 28. Tumour tissues were processed as described under "In vivo biodistribution studies", and the lungs and hearts were embedded in paraffin and processed for haematoxylin and eosin (H\&E) staining (described below). Tumours were immunostained as described above. CD206 was detected using rat anti-mouse CD206 (dilution 1/200), CD8 using rat anti-mouse CD8 (dilution 1/75 Biolegend, cat. number 100701), FOXP3 using rat anti-mouse FOXP3 (dilution 1/75, Biolegend, cat number 126401) as primary antibodies, Alexa Fluor ${ }^{\circledR}$ goat anti-rat 647 (dilution 1/300 for CD206 and 1/200 for CD8, FOXP3, was used as a secondary antibody for all markers. Slides were counterstained 
833 with DAPI $(1 \mu \mathrm{g} / \mathrm{mL}$ in PBS) and imaged using a Zeiss confocal microscope with a 10x

834 objective. All five tumours from each group were included in the IF analysis and at least three

835 images per mouse per group were included. Fluorescent signal intensity was calculated using

836 the ImageJ programme; to account for different amounts of tissue in the different images, only

837 the area containing tissue was selected and the "mean signal intensity" given by the programme

838 taken (total integrated intensity divided by the selected area). For this analysis, at least three

839 images per tumour were included.

840

$841 \quad$ H\&E staining in paraffin-embedded formalin-fixed tissues

842 For H\&E staining, $2 \mu \mathrm{m}$ sections were cut from paraffin-embedded blocks. Slides were

843 warmed at $60^{\circ} \mathrm{C}$ for $2 \mathrm{~min}$ before deparaffinising using xylene ( $\left.3 \times 2 \mathrm{~min}, 1 \mathrm{x} 1 \mathrm{~min}\right)$ followed

844 by $100 \%$ ethanol washes $(3 \times 1 \mathrm{~min}), 80 \%$ ethanol wash $(1 \times 1 \mathrm{~min})$ followed by $1 \mathrm{~min}$ wash in

845 water. Slides were first incubated with ST-1 HemaLast for 30 s, followed by ST-2

846 Haematoxylin for $5 \mathrm{~min}$ after which slides were washed in water for $2 \mathrm{~min}$. Then, ST-3

847 Differentiator was added for $45 \mathrm{~s}$, and slides were washed in water for $1 \mathrm{~min}$. Next, ST-4 Bluing

848 Agent was added (1 $\mathrm{min}$ ), washed for $1 \mathrm{~min}$ in water followed by 1 min incubation in $80 \%$

849 ethanol, after which ST-5 Eosin was added and incubated for 1 min. For rehydration,

850 incubations in $100 \%$ ethanol $(2 \times 30 \mathrm{~s}, 1 \times 2 \mathrm{~min})$ were carried out and finished with incubations

851 in xylene ( $2 \times 2 \mathrm{~min})$. All washes were carried out in tap water. H\&E stainings were performed

852 in Tartu University Hospital by pathologists using Leica staining automat and ST Infinity H\&E

853 Staining System (Leica, cat. 38016998). Stained lung sections were scanned using a slide

854 scanner (Leica SCN400) and 20x zoom. Images were analysed using the QuPath programme

855 (version 0.1 .2$)^{101}$. Five levels $\sim 1 \mathrm{~mm}$ apart were used for each mouse to obtain comprehensive

856 pulmonary metastases profile. Stained heart sections were also scanned using a slide scanner

857 and analysed with the QuPath programme. Tartu University Hospital pathologists assessed

858 cardiotoxicity in hearts and pulmonary metastases.

860 Analysis of CD31 expression and blood vessel count

861 CD31 expression after treating orthotopic TNBC tumours with OximUNO, St-PGA-DOX, or

862 DOX was detected using rat anti-mouse CD31 (dilution 1/100, BD Biosciences, cat. 553370)

863 and Alexa Fluor ${ }^{\circledR} 546$ goat anti-rat (dilution 1/200, Invitrogen, cat. A11081) was used as the

864 secondary antibody. Slides were counterstained with DAPI $(1 \mu \mathrm{g} / \mathrm{mL}$ in PBS $)$ and imaged using

865 Zeiss confocal microscope with a 10x objective. CD31 expression was calculated using ImageJ 
866 and mean signal per field as described under "OximUNO therapy in orthotopic TNBC",

867 including at least five images per mouse per group, $\mathrm{N}=5$ mice per group. The blood vessel

868 count was calculated from the same images using ImageJ as follows: the image was changed

869 to an 8-bit image, threshold (Triangle algorithm with modifications to account for as much

870 actual CD31 signal as possible) was added, and particles analysed. At least three images per

871 mouse per group were included in the analysis, $\mathrm{N}=5$ mice per group.

\section{OximUNO treatment of experimental metastasis of TNBC}

$8742 \times 10^{5} 4 \mathrm{~T} 1$ cells in $100 \mu \mathrm{L}$ of PBS were i.v. injected into the tail vein of 8-12-week-old female

875 Balb/c mice. Treatment with OximUNO, St-PGA-DOX, or DOX began on day four p.i.; each 876 group comprised six mice. Doses of different compounds were calculated based on DOX 877 (2mg/kg): DOX: $39.5 \mu \mathrm{g} / 0.5 \mathrm{~mL}$ PBS; St-PGA-DOX: $774.5 \mu \mathrm{g} / 0.5 \mathrm{~mL}$ PBS; OximUNO: 704 $878 \mu \mathrm{g} / 0.5 \mathrm{~mL}$ PBS. Mouse bodyweight was monitored every other day. A total of six injections 879 were carried out every other day. The final injection was on day 12, and all animals were 880 sacrificed on day 18 using anaesthetic overdose and perfusion with PBS. Three right lungs 881 from each group were analysed with flow cytometry (FC), and three full lungs and three left 882 lungs from each group were frozen into blocks using OCT. Frozen lung tissues were 883 cryosectioned as described earlier, fixed with cold 4\% PFA (CD206) or acetone (for CD8 and 884 FOXP3), and stained as described in the following section. Immunofluorescent stainings were 885 performed using the same markers and antibodies as shown in the "OximUNO treatment in 886 orthotopic TNBC" section.

\section{GFP staining and imaging}

889 Six lungs from each group were frozen in OCT. Ten $\mu \mathrm{m}$ sections were cut, and slides were 890 kept at $-20^{\circ} \mathrm{C}$ until ready to use. Slides were taken out of the freezer at least 30 min before 891 staining. For staining, slides were fixed with 4\% PFA for $10 \mathrm{~min}$ at room temperature, washed 892 with PBS for $10 \mathrm{~min}$ at room temperature, counterstained using DAPI ( $1 \mu \mathrm{g} / \mathrm{mL}$ in PBS) for 5 $893 \mathrm{~min}$ at room temperature, washed $3 \times 4$ min with PBS and finally mounted using mounting 894 medium. Permeabilisation was not used in this step to improve GFP visualisation. GFP was 895 visualised using its native fluorescence. Slides were imaged using Olympus confocal 896 microscope (FV1200MPE) with a 10x objective. 
899 Lungs from each group were imaged using Illumatool Bright Light System LT-9900

900 (LightTool's Research) in the green channel to visualise the fluorescent signal 901 macroscopically, and a photograph of each lung was taken. The total GFP signal of each lung 902 was quantified using the ImageJ programme using the "IntDen" value.

903

\section{Flow cytometry analysis}

905 Three mice were sacrificed using anaesthetic overdose, perfused with PBS and right lung 906 tissues were placed in cold RPMI-1640 medium supplemented with 2\% v/v FBS. Lungs were 907 cut into small pieces on ice in a solution containing collagenase IV (160 U/mL, Gibco 908 cat.17104019)/dispase (0.6 U/mL, Gibco, cat. 17105-041)/DNase I (15 U/mL; AppliChem, cat. 909 A3778) mixture. To obtain a single-cell suspension, lung pieces were incubated in $10 \mathrm{~mL}$ of 910 the same mixture at $37{ }^{\circ} \mathrm{C}$ on a rotating platform for 45-60 min, pipetting every $10 \mathrm{~min}$ to 911 improve digestion. The cells were washed with $5 \mathrm{~mL}$ of RB ("running buffer": $4 \mathrm{~mL} 0.5 \mathrm{M}$ 912 EDTA, $100 \mathrm{~mL}$ v/v FBS in $1 \mathrm{~L}$ of PBS), centrifuged $\left(350 \mathrm{~g}, 7 \mathrm{~min}, 4^{\circ} \mathrm{C}\right)$, and red blood cells 913 were lysed with $3 \mathrm{~mL}$ of ammonium-chloride-potassium lysing buffer (ACK) at room 914 temperature. Ten $\mathrm{mL}$ of RB was added, cells were centrifuged and filtered using a $100 \mu \mathrm{m}$ cell 915 strainer (Falcon, cat. 352360). Cells were counted using the brightfield mode of LUNA ${ }^{\mathrm{TM}}$

916 Automated Cell counter (Logos Biosystems). Cells were collected in RB at a concentration of $9175 \times 10^{6} / 100 \mu \mathrm{L}$, placed on a 96-well plate with conical bottom and incubated for 30 min in FcR918 blocking $2.4 \mathrm{G} 2$ hybridoma medium at $4{ }^{\circ} \mathrm{C}$. The cells were then stained for either macrophage 919 or $\mathrm{T}$ cell markers for $25-45 \mathrm{~min}$ in the dark at $+4{ }^{\circ} \mathrm{C}$, centrifuged and washed twice with RB. 920 The antibodies used are listed in Table 2. For intracellular staining of $\mathrm{T}$ cells, cells were fixed 921 using eBioscience ${ }^{\mathrm{TM}}$ FOXP3/Transcription Factor Staining Buffer Set (Thermo Fisher, cat. 00922 5523-00) according to the protocol provided. Cells were stained for 25-45 min in the dark at 923 room temperature following permeabilisation and washed twice using RB. All cells were 924 collected in $150 \mu \mathrm{L}$ of $\mathrm{RB}$, filtered through a $70 \mu \mathrm{m}$ filter (Share Group Limited) and $150 \mu \mathrm{L}$ 925 of RB was used to wash the filter. BD LSRFortessa Flow Cytometer and FCS Express 7 Flow 926 (De Novo Software) were used for analysis.

927

928

929

930 Table 2. Antibodies used in FC analysis: macrophage and T cell markers.

\begin{tabular}{|l|l|l|}
\hline$\Sigma$ & Antibody & Dilution, company \\
\hline
\end{tabular}




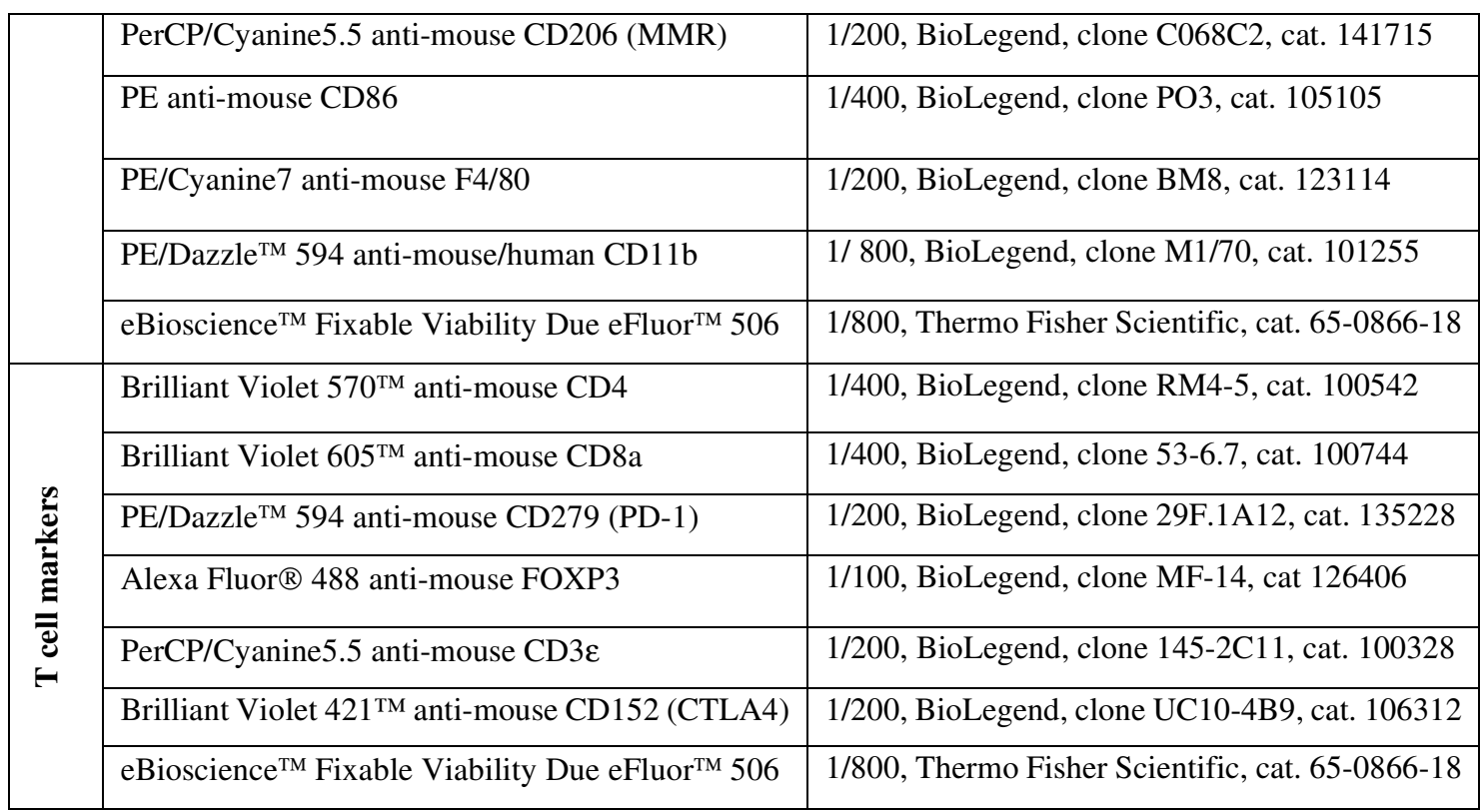

931

\section{H\&E staining on PFA-fixed cryosections}

Ten $\mu \mathrm{m}$ sections were cut from unfixed tissues in a frozen block; sections were stored at -20 ${ }^{\circ} \mathrm{C}$ until ready to use. When ready, slides were taken out of the freezer at least $30 \mathrm{~min}$ before staining. Room temperature slides were fixed with cold 4\% PFA for $10 \mathrm{~min}$ at room temperature followed by washing in PBS for $10 \mathrm{~min}$ at room temperature. After washing, slides were dipped into Mayer's haematoxylin solution (see preparation under "Reagents and Solutions") for $10 \mathrm{~s}$, followed by washing in running tap water for $5 \mathrm{~min}$. Then, slides were dipped into Eosin (5\%) solution (see preparation under "Reagents and Solutions") for $20 \mathrm{~s}$, followed by washing in running tap water for $5 \mathrm{~min}$. For rehydration, slides were placed first in $96 \%$ ethanol ( $2 \times 2 \mathrm{~min})$ followed by $100 \%$ ethanol $(2 \times 2 \mathrm{~min})$. For clearance slides were placed in RotiClear ${ }^{\circledR}$ solution (Roth, cat. A538.5) for two-times 5 min, after which slides were mounted using Eukitt ${ }^{\circledR}$ quick-hardening mounting medium (Merck, cat. 03989). Slides were scanned using Leica DM6 B microscope and Leica Aperio Versa 8 slides scanner with 20x zoom and images were analysed using the ImageScope programme (version 12.3.3).

\section{Statistical analysis}

All statistical analysis was carried out using One-Way ANOVA and Fisher LSD tests, using the Statistica programme (release 7).

\section{DATA AVAILABILITY}


952 All data needed to evaluate the conclusions on the paper are presented in the paper and/or the 953 Supplementary Information. Additional data related to the findings of this study are available 954 from the corresponding authors.

955

956

\section{REFERENCES}

958 1.Rivenbark, A. G., O’Connor, S. M. \& Coleman, W. B. Molecular and cellular

959 heterogeneity in breast cancer: challenges for personalized medicine. Am. J. Pathol. 183,

$960 \quad 1113-1124(2013)$.

961 2. Foulkes, W. D., Smith, I. E. \& Reis-Filho, J. S. Triple-Negative Breast Cancer. N. Engl. J.

$962 \quad$ Med. 363, 1938-1948 (2010).

963 3. Lehmann, B. D. et al. Identification of human triple-negative breast cancer subtypes and

964 preclinical models for selection of targeted therapies. J. Clin. Invest. 121, 2750-2767

$965 \quad$ (2011).

966 4. Garrido-Castro, A. C., Lin, N. U. \& Polyak, K. Insights into Molecular Classifications of

967 Triple-Negative Breast Cancer: Improving Patient Selection for Treatment. Cancer

968 Discov. 9, 176-198 (2019).

969 5. Pardoll, D. M. The blockade of immune checkpoints in cancer immunotherapy. Nat. Rev.

$970 \quad$ Cancer 12, 252-264 (2012).

971 6. Esfahani, K. et al. A Review of Cancer Immunotherapy: From the Past, to the Present, to 972 the Future. Curr. Oncol. 27, 87-97 (2020).

973 7. Adams, S. et al. Current Landscape of Immunotherapy in Breast Cancer: A Review. JAMA $974 \quad$ Oncol. 5, 1205-1214 (2019).

975 8. Gong, J., Chehrazi-Raffle, A., Reddi, S. \& Salgia, R. Development of PD-1 and PD-L1

976 inhibitors as a form of cancer immunotherapy: a comprehensive review of registration

977 trials and future considerations. J. Immunother. Cancer 6, 8 (2018). 
978 9. Schmid, P. et al. Atezolizumab and Nab-Paclitaxel in Advanced Triple-Negative Breast

979 Cancer. N. Engl. J. Med. 379, 2108-2121 (2018).

980 10. Schmid, P. et al. Atezolizumab plus nab-paclitaxel as first-line treatment for

981 unresectable, locally advanced or metastatic triple-negative breast cancer (IMpassion130):

982 updated efficacy results from a randomised, double-blind, placebo-controlled, phase 3

983 trial. Lancet Oncol. 21, 44-59 (2020).

984 11. Marra, A., Viale, G. \& Curigliano, G. Recent advances in triple negative breast

985 cancer: the immunotherapy era. BMC Med. 17, 90 (2019).

986 12. Mori, H. et al. The combination of PD-L1 expression and decreased tumor-infiltrating

987 lymphocytes is associated with a poor prognosis in triple-negative breast cancer.

$988 \quad$ Oncotarget 8, 15584-15592 (2017).

989 13. Mittendorf, E. A. et al. PD-L1 Expression in Triple-Negative Breast Cancer. Cancer $990 \quad$ Immunol. Res. 2, 361-370 (2014).

991 14. Socinski, M. A. et al. Atezolizumab for First-Line Treatment of Metastatic

992 Nonsquamous NSCLC. N. Engl. J. Med. 378, 2288-2301 (2018).

993 15. Adams, S. et al. Patient-reported outcomes from the phase III IMpassion130 trial of 994 atezolizumab plus nab-paclitaxel in metastatic triple-negative breast cancer. Ann. Oncol. $995 \quad 31,582-589(2020)$.

996 16. Fecher, L. A., Agarwala, S. S., Hodi, F. S. \& Weber, J. S. Ipilimumab and Its 997 Toxicities: A Multidisciplinary Approach. The Oncologist 18, 733-743 (2013).

998 17. Hunter, G., Voll, C. \& Robinson, C. A. Autoimmune inflammatory myopathy after 999 treatment with ipilimumab. Can. J. Neurol. Sci. J. Can. Sci. Neurol. 36, 518-520 (2009).

1000 18. Maker, A. V. et al. Tumor Regression and Autoimmunity in Patients Treated With 1001 Cytotoxic T Lymphocyte-Associated Antigen 4 Blockade and Interleukin 2: A Phase I/II 1002 Study. Ann. Surg. Oncol. 12, 1005-1016 (2005). 
1003 19. Phan, G. Q. et al. Cancer regression and autoimmunity induced by cytotoxic T

1004 lymphocyte-associated antigen 4 blockade in patients with metastatic melanoma. Proc.

1005 Natl. Acad. Sci. U. S. A. 100, 8372-8377 (2003).

1006 20. Cretella, D. et al. Pre-treatment with the CDK4/6 inhibitor palbociclib improves the 1007 efficacy of paclitaxel in TNBC cells. Sci. Rep. 9, 13014 (2019).

1008 21. Arola, O. J. et al. Acute Doxorubicin Cardiotoxicity Involves Cardiomyocyte 1009 Apoptosis. Cancer Res. 60, 1789-1792 (2000).

1010 22. Zhang, S. et al. Identification of the molecular basis of doxorubicin-induced 1011 cardiotoxicity. Nat. Med. 18, 1639-1642 (2012).

1012 23. Keklikoglou, I. et al. Chemotherapy elicits pro-metastatic extracellular vesicles in 1013 breast cancer models. Nat. Cell Biol. 21, 190-202 (2019).

1014 24. Hughes, R. et al. Perivascular M2 Macrophages Stimulate Tumor Relapse after 1015 Chemotherapy. Cancer Res. 75, 3479-3491 (2015).

$101625 . \quad$ Lewis, C. E. \& Pollard, J. W. Distinct Role of Macrophages in Different Tumor 1017 Microenvironments. Cancer Res. 66, 605-612 (2006).

1018 26. Peranzoni, E. et al. Macrophages impede CD8 T cells from reaching tumor cells and 1019 limit the efficacy of anti-PD-1 treatment. Proc. Natl. Acad. Sci. 115, E4041-E4050 $1020 \quad$ (2018).

1021 27. Neubert, N. J. et al. T cell-induced CSF1 promotes melanoma resistance to PD1 1022 blockade. Sci. Transl. Med. 10, eaan3311 (2018).

1023 28. Daurkin, I. et al. Tumor-Associated Macrophages Mediate Immunosuppression in the 1024 Renal Cancer Microenvironment by Activating the 15-Lipoxygenase-2 Pathway. Cancer 1025 Res. 71, 6400-6409 (2011). 
1026 29. Gok Yavuz, B. et al. Cancer associated fibroblasts sculpt tumour microenvironment 1027 by recruiting monocytes and inducing immunosuppressive PD-1+ TAMs. Sci. Rep. 9, 3172 1028 (2019).

1029 30. Pathria, P., Louis, T. L. \& Varner, J. A. Targeting Tumor-Associated Macrophages in $1030 \quad$ Cancer. Trends Immunol. 40, 310-327 (2019).

1031 31. DeNardo, D. G. et al. Leukocyte Complexity Predicts Breast Cancer Survival and 1032 Functionally Regulates Response to Chemotherapy. Cancer Discov. 1, 54-67 (2011).

1033 32. Mancini, V. S. B. W., Pasquini, J. M., Correale, J. D. \& Pasquini, L. A. Microglial 1034 modulation through colony-stimulating factor-1 receptor inhibition attenuates 1035 demyelination. Glia 67, 291-308 (2019).

1036 33. Lee, S., Shi, X. Q., Fan, A., West, B. \& Zhang, J. Targeting macrophage and 1037 microglia activation with colony stimulating factor 1 receptor inhibitor is an effective 1038 strategy to treat injury-triggered neuropathic pain. Mol. Pain 14, 1744806918764979 $1039 \quad$ (2018).

1040 34. Bissinger, S. et al. Macrophage depletion induces edema through release of matrix1041 degrading proteases and proteoglycan deposition. Sci. Transl. Med. 13, eabd4550 (2021).

1042 35. Wesolowski, R. et al. Phase Ib study of the combination of pexidartinib (PLX3397), a 1043 CSF-1R inhibitor, and paclitaxel in patients with advanced solid tumors. Ther. Adv. Med.

$1044 \quad$ Oncol. 11, 1758835919854238 (2019).

1045 36. Papadopoulos, K. P. et al. First-in-Human Study of AMG 820, a Monoclonal Anti1046 Colony-Stimulating Factor 1 Receptor Antibody, in Patients with Advanced Solid Tumors. 1047 Clin. Cancer Res. 23, 5703-5710 (2017).

1048 37. Kitamura, T. et al. Monocytes Differentiate to Immune Suppressive Precursors of 1049 Metastasis-Associated Macrophages in Mouse Models of Metastatic Breast Cancer. Front. $1050 \quad$ Immunol. 8, (2018). 
1051 38. Madsen, D. H. et al. Tumor-Associated Macrophages Derived from Circulating

1052 Inflammatory Monocytes Degrade Collagen through Cellular Uptake. Cell Rep. 21, 3662$1053 \quad 3671(2017)$.

1054 39. Ishihara, D. et al. Wiskott-Aldrich Syndrome Protein Regulates Leukocyte-Dependent 1055 Breast Cancer Metastasis. Cell Rep. 4, 429-436 (2013).

1056 40. Karousou, E. et al. Collagen VI and Hyaluronan: The Common Role in Breast 1057 Cancer. BioMed Res. Int. 2014, 1-10 (2014).

1058 41. Scodeller, P. et al. Precision Targeting of Tumor Macrophages with a CD206 Binding 1059 Peptide. Sci. Rep. 7, 14655 (2017).

1060 42. Lepland, A. et al. Targeting Pro-Tumoral Macrophages in Early Primary and 1061 Metastatic Breast Tumors with the CD206-Binding mUNO Peptide. Mol. Pharm. 17, $1062 \quad 2518-2531(2020)$.

1063 43. Asciutto, E. K. et al. Phage-Display-Derived Peptide Binds to Human CD206 and 1064 Modeling Reveals a New Binding Site on the Receptor. J. Phys. Chem. B 123, 1973-1982 1065 (2019).

1066 44. Figueiredo, P. et al. Peptide-guided resiquimod-loaded lignin nanoparticles convert 1067 tumor-associated macrophages from M2 to M1 phenotype for enhanced chemotherapy. 1068 Acta Biomater. 133, 231-243 (2021).

1069 45. Jameson, B. et al. Expression of DC-SIGN by Dendritic Cells of Intestinal and 1070 Genital Mucosae in Humans and Rhesus Macaques. J. Virol. 76, 1866-1875 (2002).

1071 46. Conniot, J. et al. Immunization with mannosylated nanovaccines and inhibition of the 1072 immune-suppressing microenvironment sensitizes melanoma to immune checkpoint 1073 modulators. Nat. Nanotechnol. 14, 891-901 (2019). 
1074 47. Duro-Castano, A. et al. Well-Defined Star-Shaped Polyglutamates with Improved

1075 Pharmacokinetic Profiles As Excellent Candidates for Biomedical Applications. Mol.

1076 Pharm. 12, 3639-3649 (2015).

1077 48. Arroyo-Crespo, J. J. et al. Tumor microenvironment-targeted poly-L-glutamic acid-

1078 based combination conjugate for enhanced triple negative breast cancer treatment.

1079 Biomaterials 186, 8-21 (2018).

1080 49. Duro-Castano, A. et al. Polyglutamic acid-based crosslinked doxorubicin nanogels as 1081 an anti-metastatic treatment for triple negative breast cancer. J. Controlled Release 332, $1082 \quad 10-20(2021)$.

1083 50. Arroyo-Crespo, J. J. et al. Anticancer Activity Driven by Drug Linker Modification in 1084 a Polyglutamic Acid-Based Combination-Drug Conjugate. Adv. Funct. Mater. 28, $10851800931(2018)$.

1086 51. Shaffer, S. A. et al. In vitro and in vivo metabolism of paclitaxel poliglumex: 1087 identification of metabolites and active proteases. Cancer Chemother. Pharmacol. 59, $1088 \quad 537-548(2007)$.

1089 52. Gordon, S. R. et al. PD-1 expression by tumour-associated macrophages inhibits 1090 phagocytosis and tumour immunity. Nature 545, 495-499 (2017).

1091 53. Zhang, M. et al. Anti-CD47 Treatment Stimulates Phagocytosis of Glioblastoma by 1092 M1 and M2 Polarized Macrophages and Promotes M1 Polarized Macrophages In Vivo. $1093 \quad$ PLOS ONE 11, e0153550 (2016).

1094 54. Simon-Gracia, L. et al. Bifunctional Therapeutic Peptides for Targeting Malignant B 1095 Cells and Hepatocytes: Proof of Concept in Chronic Lymphocytic Leukemia. Adv. Ther. 3, $10962000131(2020)$. 
1097 55. MPD: Phenotype strain survey measures: alanine aminotransferase.

1098 https://phenome.jax.org/search/details/ssmeasures?searchterm=alanine+aminotransferase+ 1099 \&ontavail=2.

1100 56. BALB/c Mouse | Charles River Laboratories. https://www.criver.com/products-

$1101 \quad$ services/find-model/balbc-mouse?region=3616.

1102 57. Cassetta, L. \& Kitamura, T. Targeting Tumor-Associated Macrophages as a Potential 1103 Strategy to Enhance the Response to Immune Checkpoint Inhibitors. Front. Cell Dev. Biol. 1104 0, (2018).

1105 58. Santoni, M. et al. Triple negative breast cancer: Key role of Tumor-Associated 1106 Macrophages in regulating the activity of anti-PD-1/PD-L1 agents. Biochim. Biophys. Acta 1107 BBA - Rev. Cancer 1869, 78-84 (2018).

1108 59. Rodell, C. B. et al. TLR7/8-agonist-loaded nanoparticles promote the polarization of 1109 tumour-associated macrophages to enhance cancer immunotherapy. Nat. Biomed. Eng. 2, $1110 \quad 578-588(2018)$.

1111 60. Loeuillard, E. et al. Targeting tumor-associated macrophages and granulocytic 1112 myeloid-derived suppressor cells augments PD-1 blockade in cholangiocarcinoma. J. Clin. 1113 Invest. 130, 5380-5396 (2020).

1114 61. Choo, Y. W. et al. M1 Macrophage-Derived Nanovesicles Potentiate the Anticancer 1115 Efficacy of Immune Checkpoint Inhibitors. ACS Nano 12, 8977-8993 (2018).

1116 62. Arlauckas, S. P. et al. Arg1 expression defines immunosuppressive subsets of tumor1117 associated macrophages. Theranostics 8, 5842-5854 (2018).

1118 63. Landry, A. P., Balas, M., Alli, S., Spears, J. \& Zador, Z. Distinct regional ontogeny 1119 and activation of tumor associated macrophages in human glioblastoma. Sci. Rep. 10, $112019542(2020)$. 
1121 64. Zheng, X. et al. Spatial Density and Distribution of Tumor-Associated Macrophages

1122 Predict Survival in Non-Small Cell Lung Carcinoma. Cancer Res. 80, 4414-4425 (2020).

1123 65. Etzerodt, A. et al. Specific targeting of CD163+ TAMs mobilizes inflammatory

1124 monocytes and promotes T cell-mediated tumor regression. J. Exp. Med. 216, 2394-2411 1125 (2019).

1126 66. Puig-Kröger, A. et al. Folate Receptor $\beta$ Is Expressed by Tumor-Associated

1127 Macrophages and Constitutes a Marker for M2 Anti-inflammatory/Regulatory

1128 Macrophages. Cancer Res. 69, 9395-9403 (2009).

1129 67. Viitala, M. et al. Immunotherapeutic Blockade of Macrophage Clever-1 Reactivates

1130 the CD8 ${ }^{+}$T-cell Response against Immunosuppressive Tumors. Clin. Cancer Res. 25,

$1131 \quad 3289-3303$ (2019).

1132 68. Linde, N. et al. Macrophages orchestrate breast cancer early dissemination and 1133 metastasis. Nat. Commun. 9, 1-14 (2018).

1134 69. Witschen, P. M. et al. Tumor Cell Associated Hyaluronan-CD44 Signaling Promotes 1135 Pro-Tumor Inflammation in Breast Cancer. Cancers 12, 1325 (2020).

1136 70. Guo, C. et al. Liposomal Nanoparticles Carrying anti-IL6R Antibody to the Tumour

1137 Microenvironment Inhibit Metastasis in Two Molecular Subtypes of Breast Cancer Mouse 1138 Models. Theranostics 7, 775-788 (2017).

1139 71. Movahedi, K. et al. Nanobody-Based Targeting of the Macrophage Mannose

1140 Receptor for Effective In Vivo Imaging of Tumor-Associated Macrophages. Cancer Res. $1141 \quad$ 72, 4165-4177 (2012).

1142 72. Azad, A. K. et al. $\gamma$-Tilmanocept, a New Radiopharmaceutical Tracer for Cancer 1143 Sentinel Lymph Nodes, Binds to the Mannose Receptor (CD206). J. Immunol. 195, 2019$2029(2015)$ 
1145 73. Jaynes, J. M., Lopez, H. W., Martin, G. R., YATES, C. \& Garvin, C. E. Peptides

1146 having anti-inflammatory properties. (2016).

1147 74. Scodeller, P. \& Asciutto, E. K. Targeting Tumors Using Peptides. Molecules 25, 808 $1148 \quad$ (2020).

1149 75. Ekladious, I., Colson, Y. L. \& Grinstaff, M. W. Polymer-drug conjugate therapeutics:

1150 advances, insights and prospects. Nat. Rev. Drug Discov. 18, 273-294 (2019).

1151 76. Duro-Castano, A., Conejos-Sánchez, I. \& Vicent, M. J. Peptide-Based Polymer

1152 Therapeutics. Polymers 6, 515-551 (2014).

1153 77. Moura, L. I. F. et al. Functionalized branched polymers: promising

1154 immunomodulatory tools for the treatment of cancer and immune disorders. Mater. Horiz.

$1155 \quad 6,1956-1973$ (2019).

1156 78. Melnyk, T., Đorđević, S., Conejos-Sánchez, I. \& Vicent, M. J. Therapeutic potential

1157 of polypeptide-based conjugates: Rational design and analytical tools that can boost

1158 clinical translation. Adv. Drug Deliv. Rev. 160, 136-169 (2020).

1159 79. Duro-Castano, A. et al. Capturing "Extraordinary" Soft-Assembled Charge-Like

1160 Polypeptides as a Strategy for Nanocarrier Design. Adv. Mater. 29, 1702888 (2017).

1161 80. Duro-Castano, A., Movellan, J. \& Vicent, M. J. Smart branched polymer drug

1162 conjugates as nano-sized drug delivery systems. Biomater. Sci. 3, 1321-1334 (2015).

1163 81. Cortez-Retamozo, V. et al. Origins of tumor-associated macrophages and neutrophils.

$1164 \quad$ Proc. Natl. Acad. Sci. 109, 2491-2496 (2012).

1165 82. Kurashige, M. et al. Origin of cancer-associated fibroblasts and tumor-associated

1166 macrophages in humans after sex-mismatched bone marrow transplantation. Commun.

1167 Biol. 1, 1-13 (2018).

1168 83. Veglia, F. \& Gabrilovich, D. I. Dendritic cells in cancer: the role revisited. Curr.

1169 Opin. Immunol. 45, 43-51 (2017). 
1170 84. Agostinis, P. et al. PHOTODYNAMIC THERAPY OF CANCER: AN UPDATE.

$1171 \quad$ CA. Cancer J. Clin. 61, 250-281 (2011).

1172 85. Cheah, H. Y. et al. Near-Infrared Activatable Phthalocyanine-Poly-L-Glutamic Acid

1173 Conjugate: Enhanced in Vivo Safety and Antitumor Efficacy toward an Effective

1174 Photodynamic Cancer Therapy. Mol. Pharm. 15, 2594-2605 (2018).

1175 86. Nguyen, V.-N., Yan, Y., Zhao, J. \& Yoon, J. Heavy-Atom-Free Photosensitizers:

1176 From Molecular Design to Applications in the Photodynamic Therapy of Cancer. Acc.

$1177 \quad$ Chem. Res. 54, 207-220 (2021).

1178 87. Tacar, O., Sriamornsak, P. \& Dass, C. R. Doxorubicin: an update on anticancer

1179 molecular action, toxicity and novel drug delivery systems. J. Pharm. Pharmacol. 65,

$1180 \quad 157-170(2013)$.

1181 88. Shan, H., Dou, W., Zhang, Y. \& Qi, M. Targeted ferritin nanoparticle encapsulating

$1182 \quad \mathrm{CpG}$ oligodeoxynucleotides induces tumor-associated macrophage M2 phenotype

1183 polarization into M1 phenotype and inhibits tumor growth. Nanoscale 12, 22268-22280

1184 (2020).

1185 89. Ramesh, A., Brouillard, A., Kumar, S., Nandi, D. \& Kulkarni, A. Dual inhibition of

1186 CSF1R and MAPK pathways using supramolecular nanoparticles enhances macrophage

1187 immunotherapy. Biomaterials 227, 119559 (2020).

1188 90. Hollmén, M. et al. G-CSF regulates macrophage phenotype and associates with poor 1189 overall survival in human triple-negative breast cancer. OncoImmunology 5, e1115177

$1190 \quad$ (2016).

1191 91. Georgoudaki, A.-M. et al. Reprogramming Tumor-Associated Macrophages by

1192 Antibody Targeting Inhibits Cancer Progression and Metastasis. Cell Rep. 15, 2000-2011 1193 (2016). 
1194 92. Zhang, F. et al. Reprogramming of profibrotic macrophages for treatment of

1195 bleomycin-induced pulmonary fibrosis. EMBO Mol. Med. 12, e12034 (2020).

1196 93. Sartor, O. et al. Lutetium-177-PSMA-617 for Metastatic Castration-Resistant

1197 Prostate Cancer. N. Engl. J. Med. 0, null (2021).

1198 94. Vikas, P., Borcherding, N. \& Zhang, W. The clinical promise of immunotherapy in 1199 triple-negative breast cancer. Cancer Manag. Res. 10, 6823-6833 (2018).

1200 95. Tian, C. et al. ff19SB: Amino-Acid-Specific Protein Backbone Parameters Trained 1201 against Quantum Mechanics Energy Surfaces in Solution. J. Chem. Theory Comput. 16, $1202 \quad 528-552(2020)$.

1203 96. AmberTools - SBGrid Consortium - Supported Software.

1204 https://sbgrid.org/software/titles/ambertools.

$120597 . \quad$ Hopkins, C. W., Le Grand, S., Walker, R. C. \& Roitberg, A. E. Long-Time-Step

1206 Molecular Dynamics through Hydrogen Mass Repartitioning. J. Chem. Theory Comput.

$1207 \quad \mathbf{1 1}, 1864-1874(2015)$.

1208 98. Berendsen, H. J. C., Postma, J. P. M., van Gunsteren, W. F., DiNola, A. \& Haak, J. R. 1209 Molecular dynamics with coupling to an external bath. J. Chem. Phys. 81, 3684-3690 1210 (1984).

$121199 . \quad$ Ryckaert, J.-P., Ciccotti, G. \& Berendsen, H. J. C. Numerical integration of the 1212 cartesian equations of motion of a system with constraints: molecular dynamics of n1213 alkanes. J. Comput. Phys. 23, 327-341 (1977).

1214 100. Ray, A. \& Dittel, B. N. Isolation of Mouse Peritoneal Cavity Cells. J. Vis. Exp. JoVE 12151488 (2010) doi:10.3791/1488.

1216 101. Bankhead, P. et al. QuPath: Open source software for digital pathology image 1217 analysis. Sci. Rep. 7, 16878 (2017).

1218 


\section{ACKNOWLEDGEMENTS}

1220 We would like to thank Stuart P. Atkinson for English editing, Merje Jakobson for performing 1221 H\&E studies on paraffin-embedded formalin-fixed tissues, Dr. Aivar Orav for in vivo toxicity 1222 study analysis and Dr. Mario Plaas for the help with the slide scanner. PS acknowledges support 1223 from the Estonian Research Council (grant: PUT PSG38 to PS) and a Feasibility fund of the 1224 University of Tartu (grant: ARENG51 to PS). AL acknowledges a PhD fellowship from the 1225 Estonian government. MJV acknowledges the support by European Research Council grants (ERC-CoG-2014-648831 "My-Nano" and ERC-PoC-2018-825798 "Polymmune"). Part of the equipment employed in this work has been funded by Generalitat Valenciana and co-financed with FEDER funds (PO FEDER of Comunitat Valenciana 2014-2020). UH acknowledges the support by EsRC Mobilitas+ grant MOBTP185. TT acknowledges the support by UT EIK grant and GMVBS0230PR.

\section{AUTHOR CONTRIBUTIONS}

1233 AL performed the in vitro and in vivo experiments, histology, immunofluorescence, flow cytometry, analysis and wrote the manuscript. AM performed chemical design, synthesis, and characterisation and edited the manuscript. UH performed flow cytometry experiments and analysis. EA, MP, and MB performed computational simulations. SD performed drug release studies and chemical characterisation. LS performed analysis and expert evaluation of $\mathrm{H} \& \mathrm{E}$ images. PP edited the manuscript and provided discussions and lab support. TT edited the manuscript, participated in the experimental design and discussions, and provided laboratory support. MJV performed chemical design, in vitro and in vivo experiment design, supervised chemical synthesis and characterisation, provided lab support, and edited the manuscript. PS supervised all the experiments, participated in their design and analysis, and edited the

\section{CORRESPONDING AUTHORS}

1246 Correspondence to Tambet Teesalu, María J. Vicent and Pablo Scodeller.

\section{ETHICS DECLARATIONS}

\section{COMPETING INTERESTS}

1250 PS and TT are inventors of patents on the mUNO peptide. MJV is an inventor of a patent on 
1252 an inventor of iRGD and CendR peptides and a shareholder of Cend Therapeutics Inc., a 1253 company that holds a license for the mUNO, iRGD and CendR peptides.

1254

1255

\section{FOR TABLE OF CONTENTS ONLY}
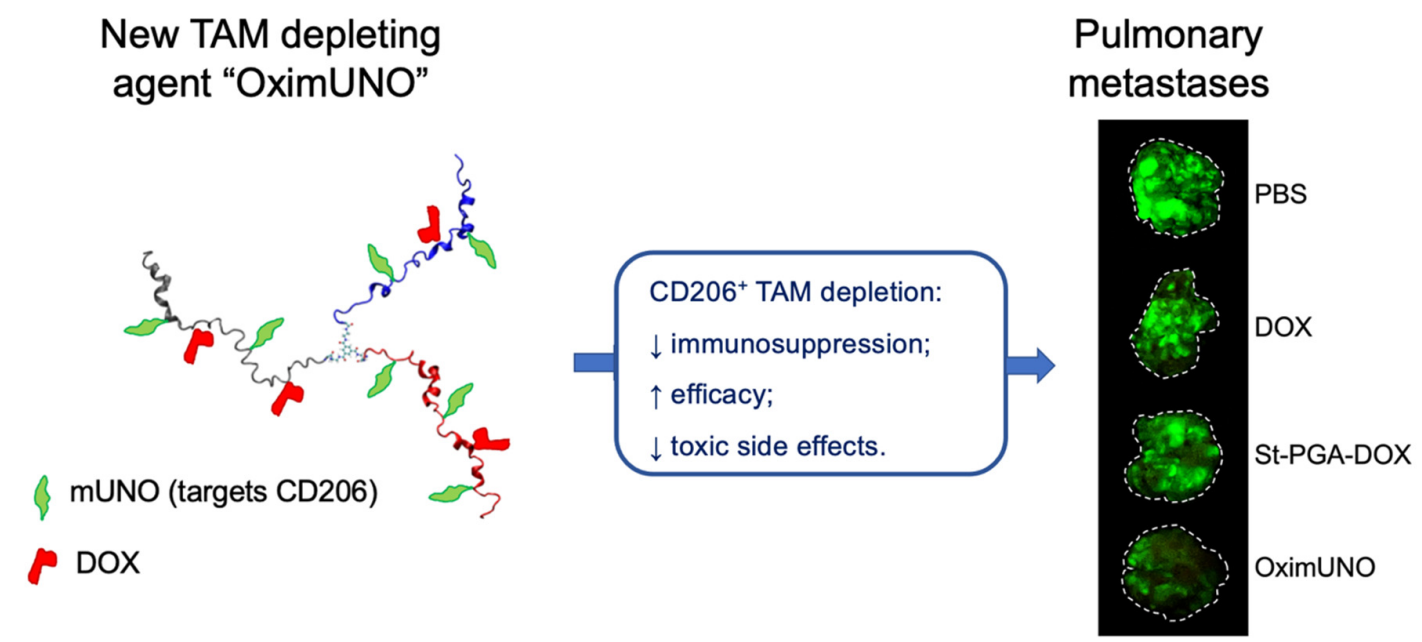

1257 


\section{Supplementary Files}

This is a list of supplementary files associated with this preprint. Click to download.

- VideoS1.gif

- OximUNOsupplementaryinformation.pdf 